\title{
Haptoglobin administration into the subarachnoid space prevents hemoglobin-induced cerebral vasospasm
}

\author{
Michael Hugelshofer, ${ }^{1}$ Raphael M. Buzzi, ${ }^{2}$ Christian A. Schaer, ${ }^{2}$ Henning Richter, ${ }^{3}$ Kevin Akeret, ${ }^{1}$ Vania Anagnostakou, ${ }^{4}$

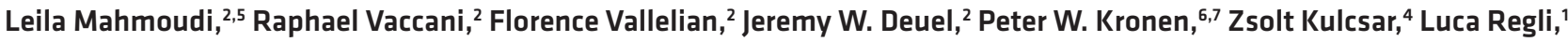 \\ Jin Hyen Baek, ${ }^{8}$ Ivan S. Pires, ${ }^{9}$ Andre F. Palmer, ${ }^{9}$ Matthias Dennler, ${ }^{3}$ Rok Humar, ${ }^{2}$ Paul W. Buehler, ${ }^{8}$ Patrick R. Kircher, ${ }^{3}$ \\ Emanuela Keller, ${ }^{10}$ and Dominik J. Schaer ${ }^{2}$ \\ 'Department of Neurosurgery, Clinical Neuroscience Center, University Hospital and University of Zurich, Zurich, Switzerland. ²Division of Internal Medicine, University Hospital of Zurich, Zurich, Switzerland. \\ ${ }^{3}$ Clinic for Diagnostic Imaging, Department of Clinical Diagnostics and Services, Vetsuisse Faculty, University of Zurich, Zurich, Switzerland. ${ }^{4}$ Department of Neuroradiology, Clinical Neuroscience Center, \\ University Hospital and University of Zurich, Zurich, Switzerland. ${ }^{5}$ Department of Chemistry and Applied Biosciences, ETH Zurich, Zurich, Switzerland. ${ }^{6}$ Veterinary Anaesthesia Services - International, \\ Winterthur, Switzerland. ' ${ }^{7}$ enter for Applied Biotechnology and Molecular Medicine (CABMM), University of Zurich, Zurich, Switzerland. ${ }^{8}$ Center of Biologics Evaluation and Research, U.S. Food and Drug \\ Administration, Silver Spring, Maryland, USA. ${ }^{9}$ William G. Lowrie Department of Chemical and Biomolecular Engineering, The Ohio State University, Columbus, Ohio, USA. ${ }^{10}$ Neurointensive Care Unit, \\ University Hospital of Zurich, Zurich, Switzerland.
}

Delayed ischemic neurological deficit (DIND) is a major driver of adverse outcomes in patients with aneurysmal subarachnoid hemorrhage (aSAH), defining an unmet need for therapeutic development. Cell-free hemoglobin that is released from erythrocytes into the cerebrospinal fluid (CSF) is suggested to cause vasoconstriction and neuronal toxicity, and correlates with the occurrence of DIND. Cell-free hemoglobin in the CSF of patients with aSAH disrupted dilatory NO signaling ex vivo in cerebral arteries, which shifted vascular tone balance from dilation to constriction. We found that selective removal of hemoglobin from patient CSF with a haptoglobin-affinity column or its sequestration in a soluble hemoglobin-haptoglobin complex was sufficient to restore physiological vascular responses. In a sheep model, administration of haptoglobin into the CSF inhibited hemoglobin-induced cerebral vasospasm and preserved vascular NO signaling. We identified 2 pathways of hemoglobin delocalization from CSF into the brain parenchyma and into the NO-sensitive compartment of small cerebral arteries. Both pathways were critical for hemoglobin toxicity and were interrupted by the large hemoglobin-haptoglobin complex that inhibited spatial requirements for hemoglobin reactions with NO in tissues. Collectively, our data show that compartmentalization of hemoglobin by haptoglobin provides a novel framework for innovation aimed at reducing hemoglobin-driven neurological damage after subarachnoid bleeding.

\section{Introduction}

Aneurysmal subarachnoid hemorrhage (aSAH) represents 5\%$10 \%$ of all strokes and has an estimated global incidence of 6.67 per 100,000 people $(1,2)$. The loss of productive life years due to aSAH is as great as that for cerebral infarction because it frequently affects patients younger than 65 years of age (3). Delayed ischemic neurological deficit (DIND) complicates aSAH in one-third of cases and is a strong risk factor for unfavorable neurological outcomes, defining an unmet need for therapeutic innovation $(4,5)$.

Within days after aSAH, red blood cells in the cerebrospinal fluid (CSF) are disintegrated by immunological and nonimmunological processes. This erythrolysis is a temporally variable process, which results in individual time profiles of cell-free hemoglobin

Authorship note: $\mathrm{MH}$ and $\mathrm{RMB}$ contributed equally to this work. Conflict of interest: MH, CAS, and DJS are inventors on a provisional patent application on the use of haptoglobin in aneurysmal subarachnoid hemorrhage (US application nos. 62/849,249 and 62/878,062)

Copyright: (ㄷ 2019, American Society for Clinical Investigation.

Submitted: May 30, 2019; Accepted: August 20, 2019; Published: October 22, 2019.

Reference information: / Clin Invest. 2019;129(12):5219-5235.

https://doi.org/10.1172/JCl130630.
$(\mathrm{Hb})$ in the CSF (6). We previously reported that elevated concentrations of $\mathrm{Hb}$ in CSF correlate with the occurrence of DIND, which suggests that cell-free $\mathrm{Hb}$ accelerates pathological processes in patients with aSAH (6). Cell-free $\mathrm{Hb}$ has been discussed for many years as a driver of damage and as a cause of vasospasm after aSAH (7-9). However, the mechanisms of toxicity remain poorly defined, and no therapeutic strategy has been proposed to specifically neutralize the devastating effects of $\mathrm{Hb}$ on the brain.

General mechanisms of cell-free $\mathrm{Hb}$ toxicity have been defined in models of systemic hemolysis, such as sickle-cell disease or after blood transfusion (10-14). The initial step of Hb toxicity is the dissociation of $\mathrm{Hb}$ tetramers $(64 \mathrm{kDa})$ into small dimers $(32 \mathrm{kDa})$ and their delocalization into tissues (15). Once delocalized, cell-free $\mathrm{Hb}$ initiates tissue damage by free-radical reactions $(16,17)$ and by NO depletion, which can cause vasoconstriction, ischemia, and cardiac failure $(10,15,18-20)$. NO-dependent signaling pathways are indispensable for functional and metabolic homeostasis of the brain (21-25). Due to the short half-life of NO in tissue, its signaling activity highly depends on the localization and compartmentalization of molecules that it can react with, such as cell-free oxyHb and deoxyHb (26-28). Disruption of endothelial NO signaling by cell-free $\mathrm{Hb}$ in cerebral arteries may 
A
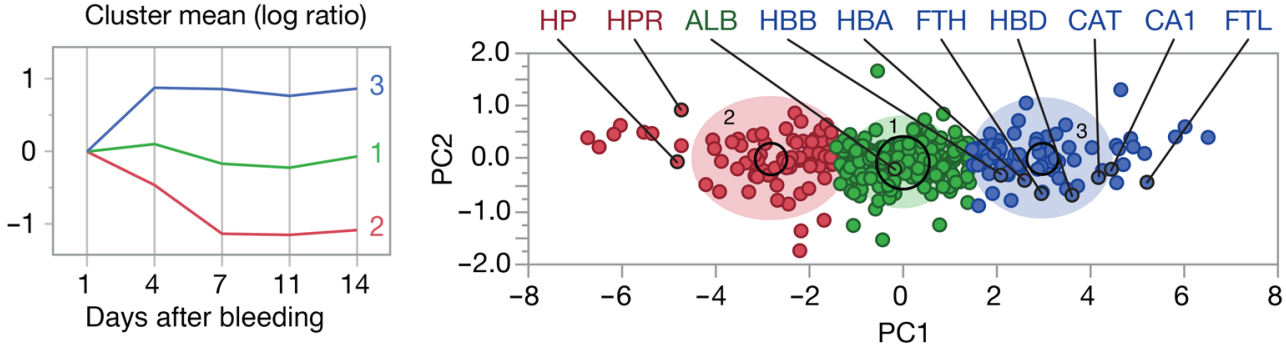

B

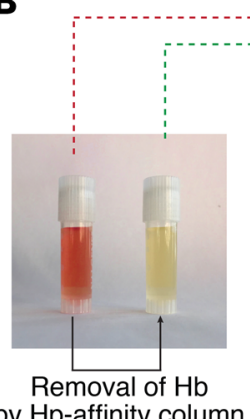
by $\mathrm{Hp}$-affinity column

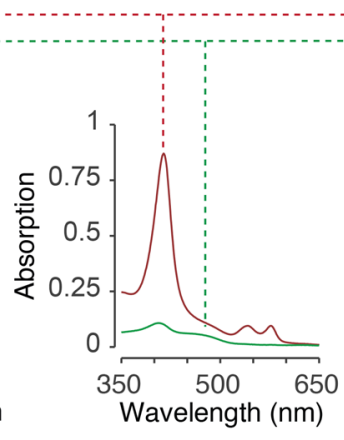

C

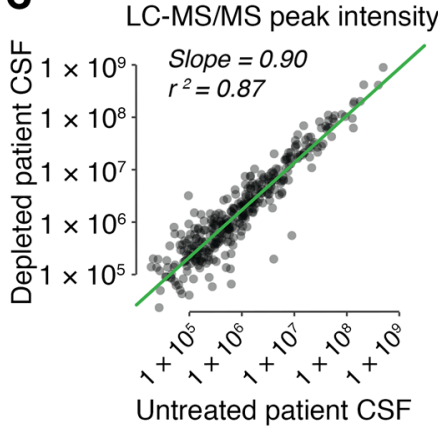

D
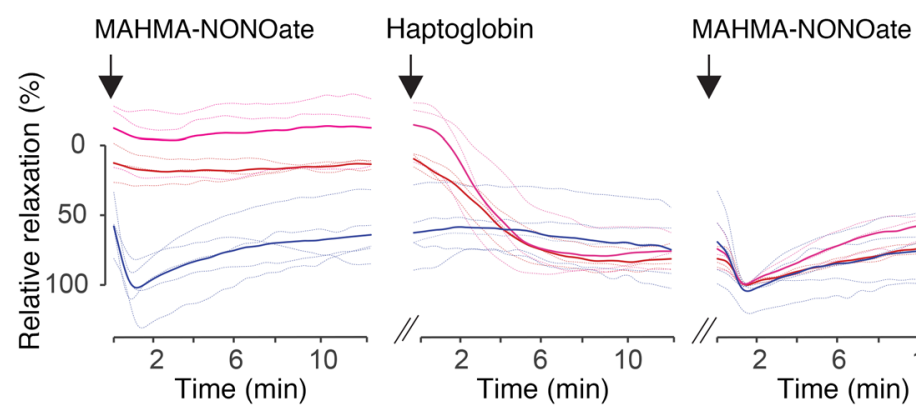

L-NIO (eNOS inhibitor)
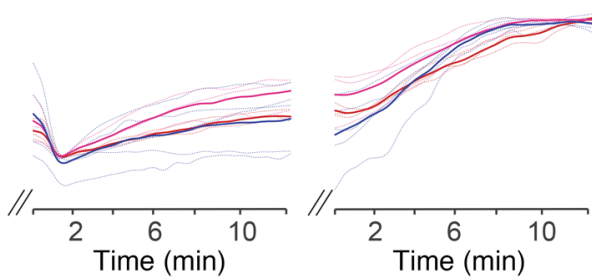

E
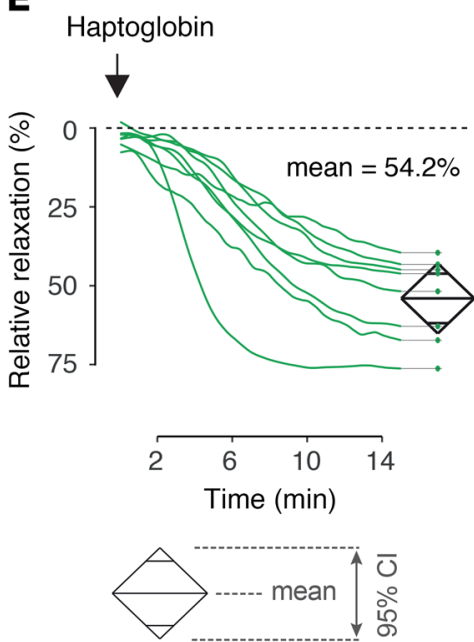

$\mathbf{F}$

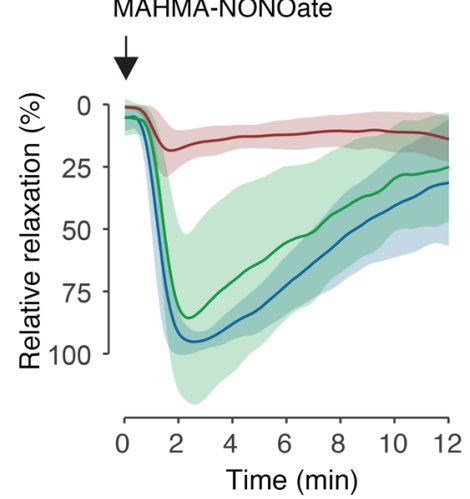

G

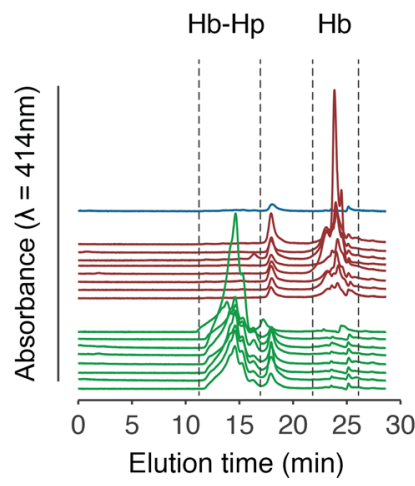

Pre-erythrolysed $\operatorname{CSF}(n=8)$

Post-erythrolysed CSF $(n=8)$

Post-erythrolysed CSF + HP $(n=8)$ 
Figure 1. Haptoglobin restores $\mathrm{Hb}$-disrupted nitric oxide signaling in cerebral arteries. (A) K-means clustering of CSF proteins of 3 patients collected at days $1,4,7,11$, and 14 after aSAH. The right panel shows a principal component analysis of the identified proteins. Cluster 1: proteins remaining unchanged. Cluster 2: proteins decreasing over time. Cluster 3: proteins increasing over time. ALB, albumin; HP, haptoglobin; HPR, haptoglobin related protein; HBB, Hb-beta; HBA, Hb-alpha; FTH, ferritin heavy-chain; HBD, Hb-delta; CAT, catalase; CA1, carbonic anhydrase; FTL, ferritin light-chain. (B) Image and absorption spectrum of a (centrifuged) patient-derived CSF sample before (red) and after (green) selective removal of $\mathrm{Hb}$. Erythrolysed CSF disrupts the dilatory NO response of porcine basilar arteries to MAHMA-NONOate. NO signaling is restored after removal of $\mathrm{Hb}$ from the CSF ( $n=12$, group mean $\pm \mathrm{SD})$. (C) Linear regression of CSF-protein ion intensities before and after passing through the haptoglobin-affinity column. (D) Tension traces of porcine basilar artery segments immersed in preerythrolysed ( $n=1$ patient; blue) and in erythrolysed ( $n=2$ patients; red/magenta) CSF from aSAH patients. Baseline and after sequential addition of MAHMA-NONOate, haptoglobin, MAHMA-NONOate, and L-NIO. Thick lines represent the mean recordings of replicate artery segments. (E) Relative changes of the steady-state tension of arteries immersed in patient-derived erythrolysed CSF after addition of haptoglobin (equimolar to cell-free $\mathrm{Hb}$ ). The diamonds represent the mean and $95 \% \mathrm{Cl}$ ( $n=8$ patient samples). (F) NO-mediated dilation of arteries immersed in CSF from aSAH patients $(n=8)$ after administration of MAHMA-NONOate. Arteries were sequentially probed in preerythrolysed CSF (blue), erythrolysed CSF (red), and after the addition of haptoglobin to the erythrolysed CSF (green). (C) Size exclusion chromatography of CSF analyzed in $\mathrm{E}$ and $\mathrm{F}$ before $(\mathrm{Hb})$ and after addition of haptoglobin ( $\mathrm{Hb}-\mathrm{Hp})$.

cause vasospasms (29-33) and may facilitate microthrombosis by disinhibiting platelet adhesion and aggregation (34-36). Within the brain parenchyma, depletion of NO may lower the threshold for pathological electrical activity, which leads to cortical spreading depolarizations $(37,38)$ and potentially dysregulates neuroinflammation $(39,40)$, eventually resulting in DIND. Despite the advanced scientific knowledge about the multifaceted pathogenesis of DIND, detection and treatment of angiographic vasospasm remains a pivotal factor in prevention of secondary neurological damage in aSAH patients $(4,41)$.

Haptoglobin is the archetypical Hb scavenger protein in vertebrates. It irreversibly binds cell-free $\mathrm{Hb}$ in the plasma, forming a large protein complex of greater than $150 \mathrm{kDa}(42)$. In systemic hemolysis, haptoglobin blocks cell-free $\mathrm{Hb}$ extravasation and attenuates oxidative tissue toxicity and cardiovascular dysregulation that is triggered by $\mathrm{Hb}$ exposure $(12,15,43-46)$. After aSAH, plasma-derived haptoglobin enters the CSF along with other plasma proteins (47). However, physiological quantities of haptoglobin cannot match the amount of cell-free $\mathrm{Hb}$ that is released by the erythrolytic process into the subarachnoid space, which results in uncontrolled cell-free $\mathrm{Hb}$ toxicity (47).

In this study, we explored whether haptoglobin could neutralize the vasoconstrictive activity of cell-free $\mathrm{Hb}$ in CSF of patients with aSAH. Additionally, we developed a sheep model to provide proof-of-concept that haptoglobin administration into the CSF can prevent cell-free $\mathrm{Hb}$-mediated toxicity in the subarachnoid space, providing a novel framework for therapeutic development.

\section{Results}

Cell-free $\mathrm{Hb}$ in the CSF of patients with aSAH is the major disruptor of arterial NO signaling. In a clinical study, we found that patients with DIND had higher cumulative cell-free $\mathrm{Hb}$ concentrations in CSF over a time period of 14 days after aSAH than patients without DIND (6). Vasospasm remains an important feature of DIND. Therefore, we wanted to define the role of cell-free $\mathrm{Hb}$ as a disruptor of vasodilatory arterial NO signaling in aSAH patients, and explore whether this toxic effect could be prevented by the $\mathrm{Hb}$-binding properties of haptoglobin.

We collected CSF samples between 1 and 14 days after subarachnoid hemorrhage from patients with aSAH and DIND. To define temporal changes in protein composition of CSF, we performed a quantitative liquid chromatography mass-spectrometry (LC-MS/ MS) proteome analysis of sequential CSF samples from 3 cases. Proteins identified by at least 2 unique peptides were classified into 3 categories by a k-means clustering analysis of the log-transformed and mean-normalized ion intensity ratios (day $\mathrm{x} /$ day 1 ) (Figure 1A and Supplemental Data Set 1; supplemental material available online with this article; https://doi.org/10.1172/JCI130630DS1). Cluster 1 contained proteins whose log ratios remained unchanged over time, such as albumin. Cluster 2 represented proteins with $\log$ ratios that decreased. This group contained haptoglobin. Cluster 3 encompassed proteins with increasing log ratios. This group included many red blood cell components, namely $\mathrm{Hb}$ and erythrocyte enzymes. This finding illustrates the delayed erythrolytic process that occurs in the subarachnoid space after aSAH, coinciding with DIND. Within cluster 3, we have also detected high concentrations of ferritin light and heavy chains, which indicates a localized adaptive macrophage response resulting in heme metabolism and iron detoxification. Endogenous haptoglobin was abundant in the initial samples but it was depleted over time. These data suggest that a large fraction of unbound $\mathrm{Hb}$ accumulates over time in the CSF of patients with aSAH.

An early clinical feature of DIND is the occurrence of vasospasms in cerebral arteries. Reactions of cell-free oxyHb $\left(\mathrm{Fe}^{2+} \mathrm{O}_{2}\right)$ and deoxyHb $\left(\mathrm{Fe}^{2+}\right)$ with the vasodilator NO may shift the balance toward vasoconstriction. To investigate this mechanism of dysregulation, we quantified the NO-mediated vasodilatory responses ex vivo, using porcine basilar arteries immersed in human CSF samples. The expected dilatory response to administration of a shortlived NO donor (MAHMA-NONOate) was suppressed in arteries immersed in CSF that was collected from a patient during DIND at day 7 after aSAH, which contained a high concentration of cellfree $\mathrm{Hb}$ (Figure 1B). The physiological vasodilatory response to MAHMA-NONOate was restored when we selectively removed cell-free $\mathrm{Hb}$ from this $\mathrm{CSF}$ sample with a haptoglobin-affinity column $(P<0.001$ for comparison of maximal dilatory responses between conditions, for each condition $n=12$ dilation responses). Analyses of the CSF by LC-MS/MS before and after Hb removal demonstrated that the concentrations of other proteins in the CSF remained mostly unchanged by the haptoglobin column (Figure 1C). This result confirmed that cell-free $\mathrm{Hb}$ is the disruptor of arterial NO signaling in the CSF of patients with aSAH. Supplemental Figure 1 shows that suppression of dilatory NO-signaling is dependent on the $\mathrm{Hb}$ concentration with complete suppression at concentrations exceeding $10 \mu \mathrm{M}$ oxyHb.

Addition of haptoglobin to patient-derived CSF restores the vascular dilatory response to intrinsic and extrinsic NO. In the next experimental step, we evaluated whether haptoglobin in solution 

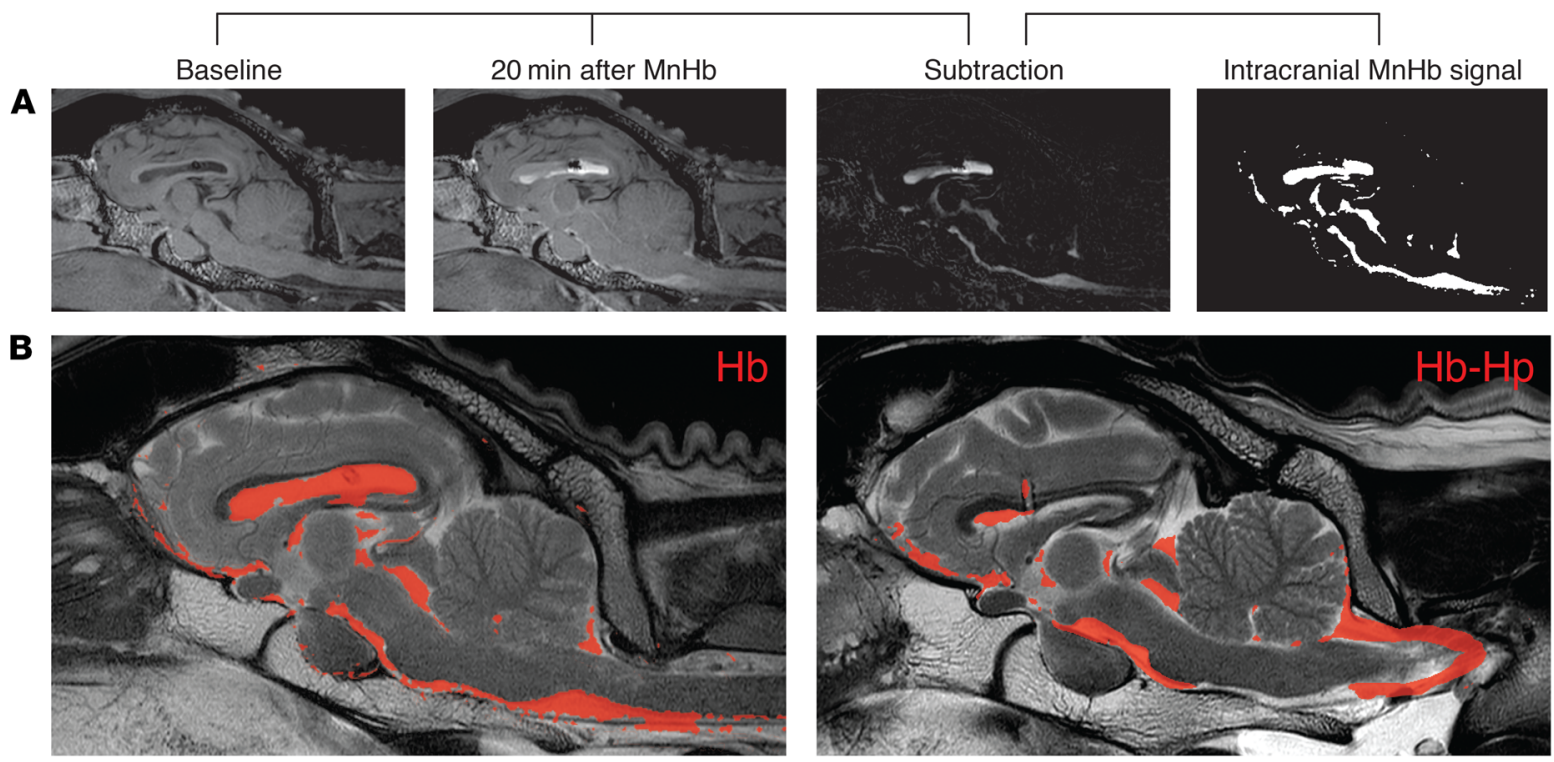

C
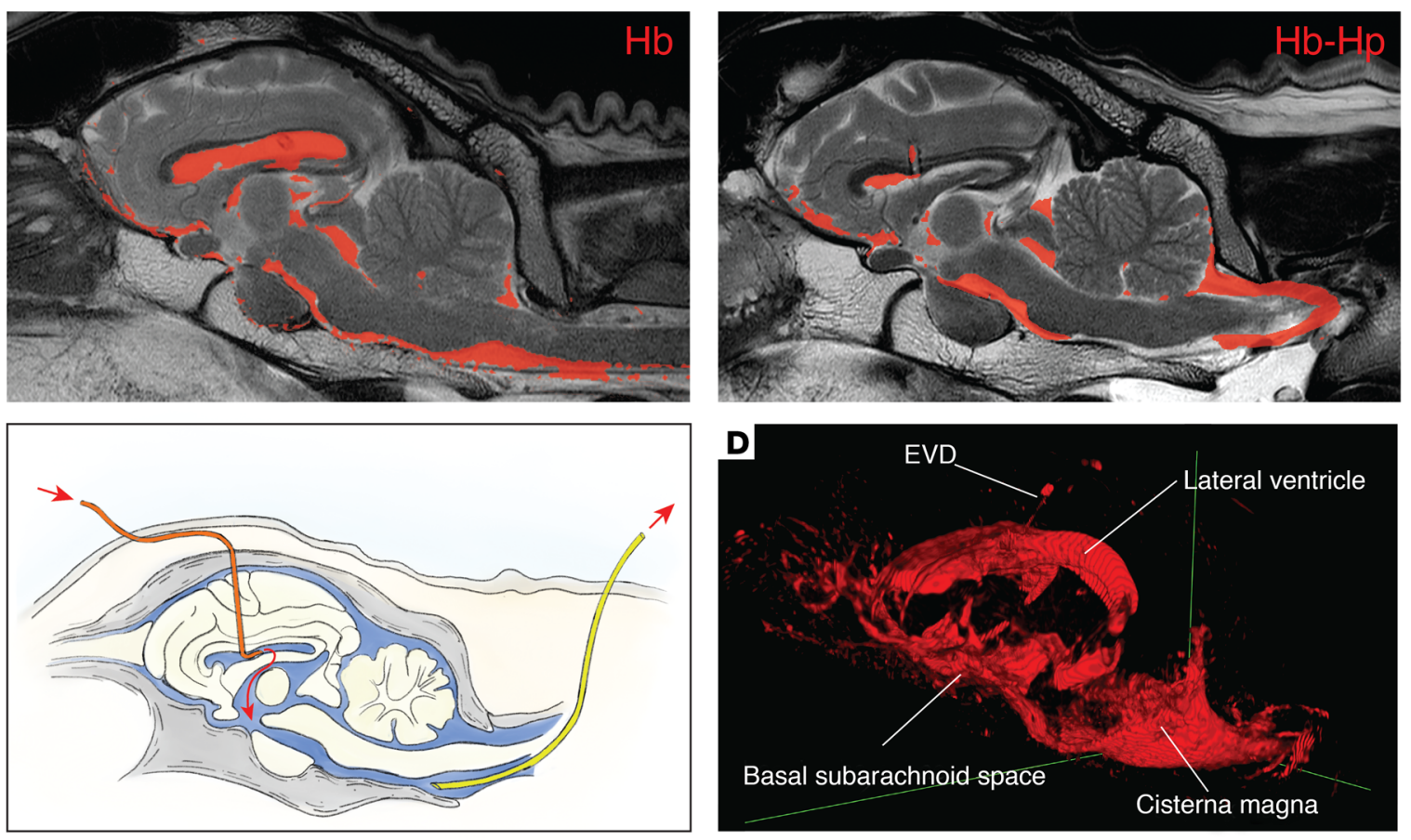

$\mathbf{E}$

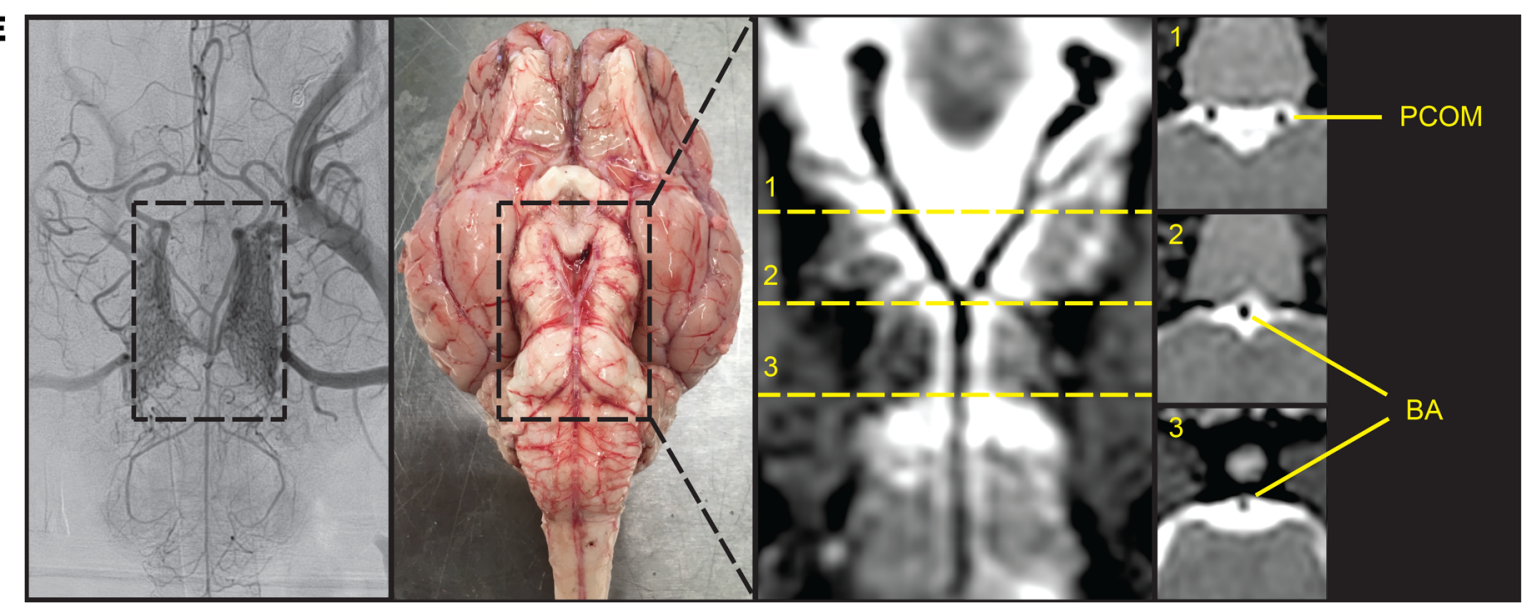

Figure 2. Distribution of hemoproteins after intraventricular infusion in sheep. (A) Sagittal reconstruction of T1-weighted MR images from a sheep before and 20 minutes after infusion of $\mathrm{MnHb}$. Subtraction of the baseline image from the image after infusion reveals the specific signal of MnHb. Binary image of the intracranial MnHb signal. (B) Overlay of the signals from MnHb (left) and MnHb-haptoglobin (right) 20 minutes after infusion on the corresponding T2-weighted anatomical MR images. (C) Schematic diagram indicating the positions of the external ventricular drain (orange) and the suboccipital subarachnoid drainage catheter (yellow). (D) Three-dimensional reconstruction of the MnHb signal 20 minutes after infusion. (E) Visualization of the circle of Willis on digital subtraction angiography (DSA), on a photograph of an anatomical specimen and on a curved multiplanar reconstruction (MPR) of a T1-weighted MR image. The bright (white) signal in the curved MPR represents the infused hemoprotein (MnHb-haptoglobin complex in this example), surrounding the posterior communicating artery (PCOM) and the basilar artery (BA), as indicated in the coronal view images. The dashed lines (labeled 1-3) in the curved MPR indicate the location of the coronal sections shown on the right. DSA images were obtained with a $\times 1.5$ magnification. 
could also restore $\mathrm{Hb}$-disrupted arterial NO signaling ex vivo (Figure 1D). In these experiments, we investigated the effect of aSAH patient-derived CSF on the intrinsic vasomotor balance of porcine basilar arteries, followed by the assessment of the dilatory response to exogenous NO, and the effect of haptoglobin addition.

We used a control CSF sample collected from a patient at day $\mathrm{O}$ after aSAH that contained no detectable cell-free $\mathrm{Hb}$ and CSF samples collected between days 4-10 from 2 patients with severe DIND, which contained cell-free $\mathrm{Hb}$ of $17.2 \mu \mathrm{M}$ and 26.8 $\mu \mathrm{M}$ oxyHb, respectively. The vascular tension traces in Figure $1 \mathrm{D}$ show that the basilar artery segments that were immersed in the erythrolysed patient-derived CSF produced a higher steady-state tension than the segments that were immersed in the nonerythrolysed control CSF, indicating that the endogenous vascular NO pool was diminished when cell-free $\mathrm{Hb}$ was present. After the administration of a MAHMA-NONOate bolus, vessels that were immersed in erythrolysed CSF did not dilate, whereas vessels immersed in control CSF responded with a physiologic relaxation. Addition of haptoglobin (40 $\mu \mathrm{M})$ to all samples did not have any effect on the tension of the artery segments that were immersed in control CSF, while the artery segments immersed in erythrolysed CSF slowly dilated until they reached the tension level of the controls. This slow dilation occurred in the absence of any exogenous NO donor, which suggests that haptoglobin restored an intrinsic vasodilatory pathway. At this point in the experiment, all arterial segments demonstrated an equal transient dilatory response to exogenous NO delivered by a bolus of MAHMA-NONOate. Haptoglobin restored the disrupted NO signaling effect of cellfree $\mathrm{Hb}$. We then inhibited endogenous NO production with the NO-synthase inhibitor L-NIO $(20 \mu \mathrm{M})$, which caused a slow contraction of all artery segments to the same steady-state level that was initially recorded with the basilar arteries exposed to erythrolysed patient-derived CSF. This contractile response reflected the loss of a similar endogenous NO pool in all artery segments.

To corroborate these observations, we further tested the ex vivo effect of haptoglobin using erythrolysed CSF collected from 8 patients between days 4-10 after aSAH containing cell-free oxyHb concentrations between $11.5 \mu \mathrm{M}$ and $49 \mu \mathrm{M}$ (mean $19 \mu \mathrm{M}$ \pm SD $12.5 \mu \mathrm{M})$. After the addition of haptoglobin, in a concentration that matched the cell-free Hb concentration in each sample, we observed a spontaneous dilation of the basilar artery segments in all patient-derived CSF samples until tensions reached approximately half of the initial values (Figure 1E). Haptoglobin also restored the vasodilatory response to exogenous NO to the level observed when using CSF that was sampled from the same 8 patients at day 0 or day 1 after aSAH, before erythrolysis occurred (Figure 1F). One-way ANOVA: $P<0.001$; Tukey-Kramer post test corrected for multiple comparison: $P<0.001$ for post-erythrolysed CSF versus the 2 other conditions and $P=0.94$ between control CSF and post-haptoglobin CSF, for each group $n=8$ dilation responses were performed. Size-exclusion chromatography (SEC) demonstrated that the addition of equimolar haptoglobin resulted in the binding of almost $100 \%$ of $\mathrm{Hb}$ in all patient samples as demonstrated by the shifted heme elution profile (Figure $1 G$ ).

Collectively, these data demonstrate that $\mathrm{Hb}$ is the main disruptor of arterial NO signaling in the CSF of patients with
aSAH, and that it remains accessible to binding and neutralization by haptoglobin.

Haptoglobin halts $\mathrm{Hb}$-induced cerebral vasospasms in sheep. To validate our approach of using haptoglobin to reverse $\mathrm{Hb}$ mediated cerebral artery vasospasms in vivo, we established a sheep ventricular infusion model. In this model, the distribution of $\mathrm{Hb}$ and $\mathrm{Hb}$-haptoglobin complexes could be visualized by T1-weighted MRI and vasospasms could be monitored by selective cerebral digital subtraction angiography (DSA). We slowly infused artificial CSF, or solutions containing $\mathrm{Hb}$, haptoglobin, or $\mathrm{Hb}$-haptoglobin complexes into the frontal horn of the left lateral ventricle (Figure 2C). The intracranial pressure was continuously monitored to avoid its uncontrolled change during the experiment (Supplemental Figure 2). Minutes after infusion, the hemoproteins appeared in the CSF, which drained from a suboccipital spinal cannula, indicating a rapid distribution from the lateral ventricle into the external CSF compartment (i.e., the subarachnoid space). The distribution of cell-free $\mathrm{Hb}$ and $\mathrm{Hb}$-haptoglobin complexes was visualized by MRI after replacing iron protoporphyrin (FePP) in the hemoproteins with manganese protoporphyrin (MnPP) (Figure 2A). The T1-weighted MRI images confirmed that the $\mathrm{Hb}(\mathrm{MnPP})$ and $\mathrm{Hb}(\mathrm{MnPP})$ haptoglobin complexes were distributed equally throughout the ventricular system, the cisterna magna, and the basal subarachnoid space (Figure 2B and Supplemental Videos 1-3). Twenty minutes after infusion, a robust MnPP signal at the base of the brain visualized the arterial circle of Willis immersed in CSF containing a high concentration of hemoproteins (Figure 2, D and E). These results confirmed that our ventricular infusion model effectively distributed $\mathrm{Hb}$ and haptoglobin into the subarachnoid space.

We then used the sheep ventricular infusion model to assess the effect of cell-free $\mathrm{Hb}$ on vascular tone in vivo (Figure 3A). Selective cerebral angiography performed after the infusion of a 5 -mL bolus of cell-free oxyHb $\left(\mathrm{HbFe}^{2+} \mathrm{O}_{2}, 5 \mathrm{mM}\right)$ into the ventricular CSF demonstrated segmental vasospasms in different arteries in angiographies recorded after 45 minutes and 60 minutes (Figure 3B and Figure 4A), which coincided with drainage of cell-free $\mathrm{Hb}$ from the subarachnoid space as recorded by SEC (Figure 3A). To test whether cell-free $\mathrm{Hb}$ in the subarachnoid space was accessible for binding and neutralization by haptoglobin, we administered a concentrated haptoglobin solution $(5 \mathrm{~mL}, 4 \mathrm{mM})$ into the lateral ventricle of a sheep previously infused with $\mathrm{Hb}$. Within minutes, $100 \%$ of cell-free $\mathrm{Hb}$ was bound to haptoglobin, forming Hb-haptoglobin complexes (as indicated by SEC); subsequently, $\mathrm{Hb}$-induced vasospasms soon resolved.

The experimental approach with sequential injection of $\mathrm{Hb}$ and haptoglobin into sheep CSF has several limitations, such as different time points in the experiment at which we evaluated vasospasms before and after haptoglobin infusion, as well as possibly heterogeneous dilution effects (i.e., the haptoglobin solution dilutes the $\mathrm{Hb}$ in $\mathrm{CSF}$ ). To overcome these limitations, we repeated the experiments with injection of a $2.5-\mathrm{mL}$ bolus of either $\mathrm{Hb}$ or $\mathrm{Hb}$-haptoglobin complexes at equal concentrations (3 $\mathrm{mM}$ heme) and visualized the cerebral vascular morphology by DSA 60 minutes after injection for quantitative analysis of vasospasms. Segmental vasospasms occurred in 4 of 4 sheep injected with $\mathrm{Hb}$, whereas no vasospasms were detected in 4 
A

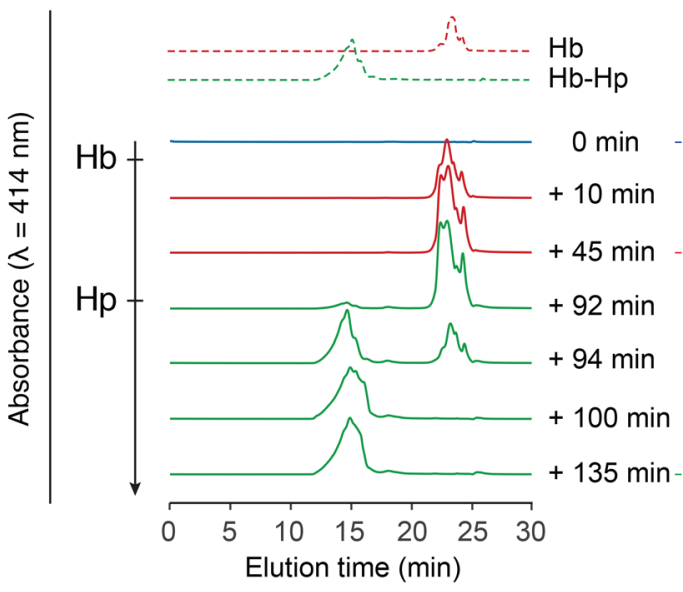

C

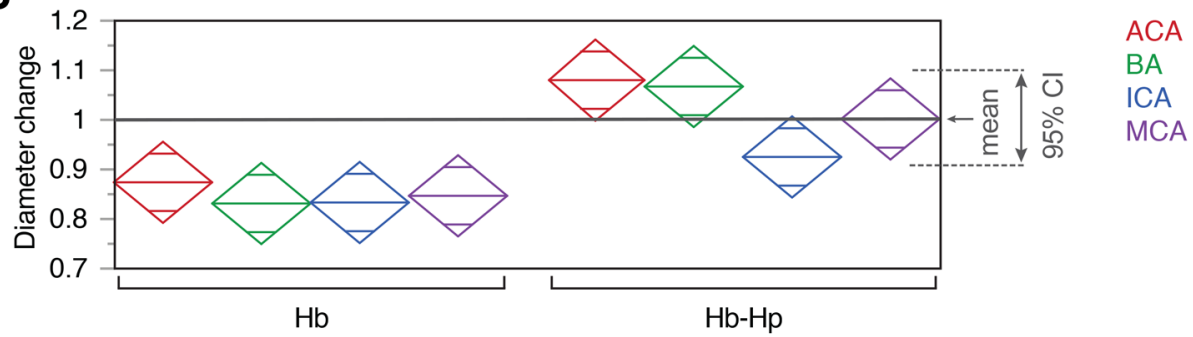

D
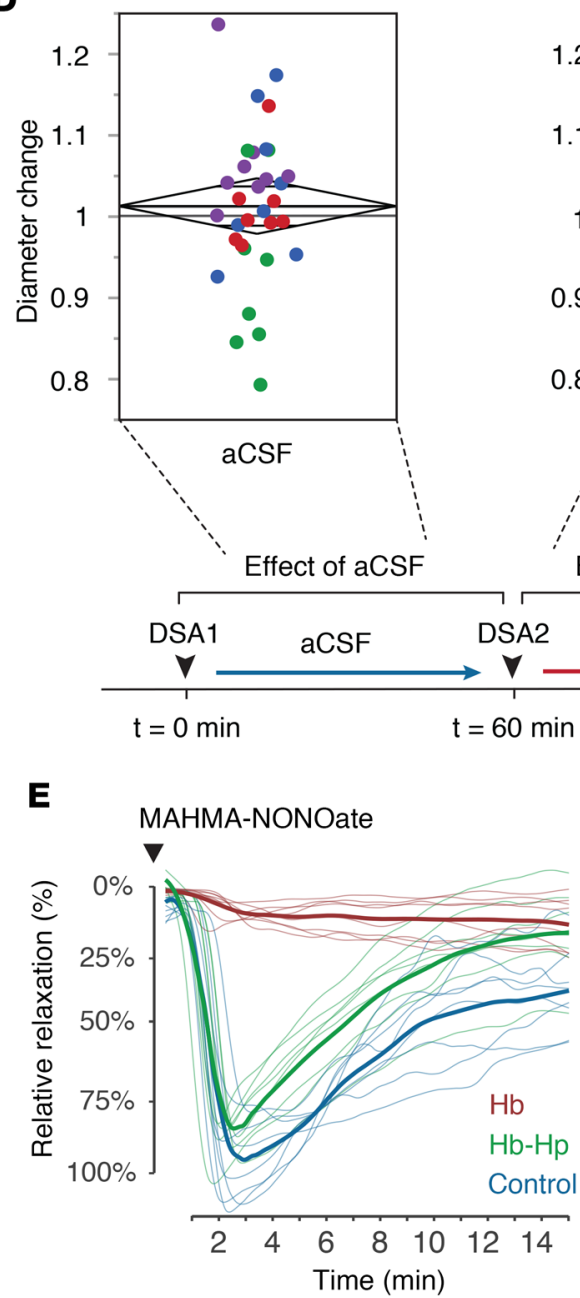

B
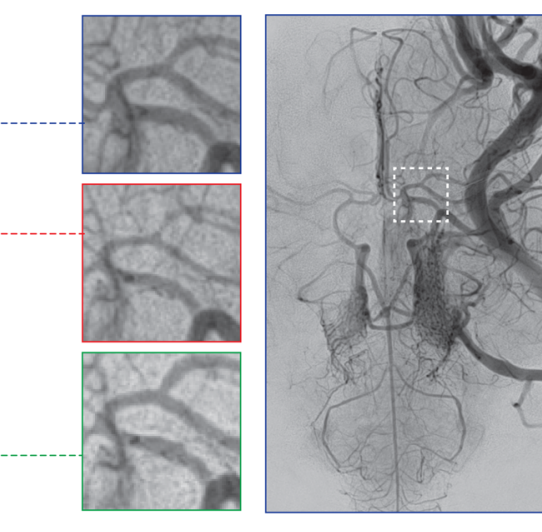

\section{F}

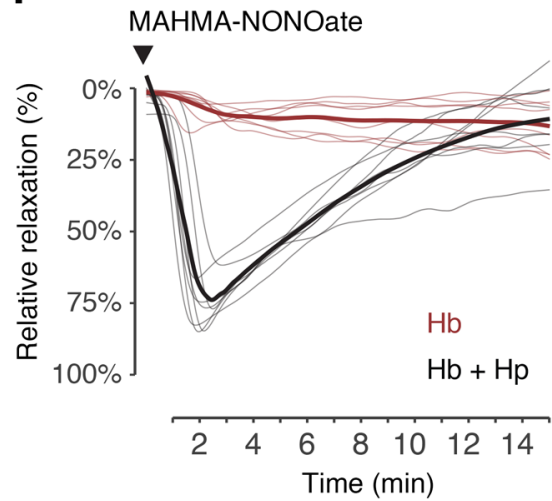

Collection of CSF for ex vivo analysis

Figure 3. Hb-induced vasospasm of cerebral arteries and protection by haptoglobin. (A) SEC elution profiles of CSF collected from the subarachnoid space at baseline (blue), after intraventricular infusion of $\mathrm{Hb}$ at $t=0$ minutes (red), and after infusion of haptoglobin at $t=90$ minutes (green). Standard elution profiles are shown on the top. (B) Angiograms of the middle cerebral artery (MCA) at baseline, 45 minutes after $\mathrm{Hb}$ infusion ( $t=45$ minutes) and 45 minutes after haptoglobin infusion ( $t=135 \mathrm{~min}$ utes). The square in the far-right image indicates the position of the zoomimages. DSA images were obtained with a $\times 1.5$ magnification. (C) Relative change in diameter of cerebral arteries $60 \mathrm{~min}$ utes after infusion of $\mathrm{Hb}$ or $\mathrm{Hb}$-haptoglobin. ACA, anterior cerebral artery; BA, basilar artery; ICA, internal carotid artery. Diamonds represent the mean and the $95 \%$ confidence interval ( $n=4$ sheep per group). (D) Cumulative analysis of the relative diameter changes of analyzed arterial segments 60 minutes after infusion of aCSF $(n=32), \mathrm{Hb}(n=16)$, or Hb-haptoglobin $(n=16)$. Colors represent vascular areas defined in $\mathbf{C}$. Group means were compared by 1-way ANOVA, $P<0.001$. The scheme depicts the experimental protocol. (E) NO-mediated relaxation profiles ( $n=8$ per condition) of porcine basilar arteries immersed in sheep CSF collected after the 60-minute posttreatment angiograms. Blue line: CSF samples after infusion of artificial CSF (CSF-heme $0 \mu \mathrm{M})$. Red line: CSF samples after infusion of $\mathrm{Hb}$ (CSF-heme 200-240 $\mu \mathrm{M}$ ). Green line: CSF samples after infusion of Hb-haptoglobin (CSF-heme 200-240 $\mu \mathrm{M}$ ). Thick lines represent the treatment group means. Dilatory responses were induced with a single bolus of MAHMA-NONOate. (F) Addition of equimolar haptoglobin to the $\mathrm{Hb}$-containing sheep CSF restored the dilatative response to MAHMA-NONOate. Red line: before haptoglobin addition, same CSF as in $\mathbf{E}$. Black line: after haptoglobin addition. Thick lines represent treatment group means of relaxation profiles $(n=8)$. 
sheep injected with Hb-haptoglobin complexes (Figure 4B). We performed a blinded and semiautomatic quantitative analysis of arterial diameters in the DSA images of 4 ex ante defined cerebral artery regions to objectify the visual impression of vasospasms (Supplemental Figure 3). We found a significant narrowing of the basilar artery and anterior and middle cerebral arteries, as well as of the cisternal internal carotid artery in Hb-infused animals compared with those in Hb-haptoglobin-infused animals (Figure 3C). Hb-induced vascular contractions became even more apparent in a pooled analysis of all arterial segments (Figure 3D). The $\mathrm{Hb}$-induced reduction in cerebral artery diameters appeared to be moderate (15\%). However, considering that the resistance (R) of a vessel is inversely proportional to the fourth power of its radius ( $\mathrm{R}$ $\left.\sim 1 / \mathrm{r}^{4}\right)$, a $15 \%$ reduction in arterial diameter doubles the vascular resistance in these segments. We found no significant change in arterial diameters after $\mathrm{Hb}$-haptoglobin infusion or after infusion of an equal volume of artificial CSF, which was performed as a general control procedure during the initial phase of each experiment (Figure 3D). These results showed that cell-free $\mathrm{Hb}$ causes vasospasms accompanied by a reduction in arterial diameters and that this was reversed by binding of $\mathrm{Hb}$ to haptoglobin.

To assess whether the recovery of $\mathrm{Hb}$-induced vasospasms by haptoglobin was related to effects on vascular NO signaling, we quantified NO-mediated arterial dilation ex vivo using porcine basilar artery segments immersed in sheep CSF sampled from the subarachnoid space of the sheep used in the previous experiments, immediately after angiography (Figure 3E). We found that CSF from Hb-infused sheep (200-240 $\mu \mathrm{M} \mathrm{Hb}$ ) completely suppressed the dilatory response to MAHMA-NONOate. In contrast, the dilatory response in CSF from $\mathrm{Hb}$-haptoglobin-infused animals (200-240 $\mu \mathrm{M} \mathrm{Hb}$-haptoglobin) was intact and not different from the physiological response recorded in CSF samples collected after the injection of artificial CSF. Finally, we explored whether haptoglobin could restore physiological responses in arteries rendered unresponsive to NO by previous immersion in CSF from $\mathrm{Hb}$-infused animals. When adding haptoglobin to such an equilibrated system at quantities that matched the concentration of cell-free $\mathrm{Hb}$, physiological responses of the cerebral arteries to NO were reestablished within minutes (Figure 3F). Comparison of the maximal vasodilatory responses revealed a significant difference between the $\mathrm{Hb}$ CSF and all other groups ( $P<0.0001, n=4$ per group). These results are in concordance with the outcome of our initial in vivo experiment in which delayed administration of haptoglobin into the ventricular CSF reverted cell-free $\mathrm{Hb}$-induced vasospasms.

Collectively, the functional data obtained in our sheep model demonstrate that haptoglobin administration into the CSF could bind cell-free $\mathrm{Hb}$ in a $\mathrm{Hb}$-haptoglobin complex restoring vascular NO signaling and cerebral artery dilation.

$\mathrm{Hb}$ and the Hb-haptoglobin complex have identical NO reaction kinetics in the subarachnoid space. The reaction of oxyHb with NO is the fundamental mechanism that causes $\mathrm{Hb}$-induced vasospasms in our model. NO reacts with oxyHb in a 3-step reaction (Figure $5 \mathrm{~A}$ and ref. 27). We have previously reported estimates for the NO reaction kinetics of oxyHb bound to haptoglobin (15). However, these experiments were performed under conditions of $\mathrm{Hb}$ excess over NO, and therefore captured only the first step of the reaction, which oxidizes oxyHb $\left(\mathrm{Fe}^{2+}\right)$ to metHb $\left(\mathrm{Fe}^{3+}\right)$, consuming $1 \mathrm{NO}$ molecule. In the current study, we compared NO reaction kinetics of cell-free $\mathrm{Hb}$ and $\mathrm{Hb}$-haptoglobin complex using an excess concentration of NO, which allows us to follow the sequence of reactions. Figure $5 \mathrm{~B}$ shows absorbance changes at $405 \mathrm{~nm}$ of $\mathrm{Hb}$ and the $\mathrm{Hb}$-haptoglobin complex after rapid mixing with NO in a stop-flow instrument. We used a glutaraldehyde polymerized $\mathrm{Hb}$ as a control compound that mimics the large size of the Hb-haptoglobin complex lacking the additional protein component of haptoglobin. The absorbance data could be best approximated by a function expressed as a sum of 3 exponentials, which mathematically supports the 3-step reaction mechanism (Supplemental Figure 4, $\mathrm{A}$ and $\mathrm{B})$. The calculated rate constants for $\mathrm{k} 1$ and $\mathrm{k} 2$ confirm that the NO reaction is very similar for all 3 hemoproteins. In another stop-flow experiment, we compared the reaction of NO with CSF that was recovered from the subarachnoid space of sheep after intraventricular infusion of $\mathrm{Hb}$ or $\mathrm{Hb}$-haptoglobin complex. This experiment demonstrates that haptoglobin does not change the biochemical reaction of $\mathrm{Hb}$ with $\mathrm{NO}$ in the subarachnoid space, as long as the hemoprotein remains accessible for NO (Supplemental Figure 4, C and D).

Large molecular size of the Hb-haptoglobin complex preserves dilatory NO signaling in porcine basilar arteries and prevents translocation of the NO-reactive complexes into the brain. In light of the very divergent effect on vascular NO signaling, the identical NOreaction kinetics of cell-free $\mathrm{Hb}$ and the $\mathrm{Hb}$-haptoglobin complex appear to be contradictory. We have therefore used the abovedescribed polymerized $\mathrm{Hb}$, which differs from cell-free $\mathrm{Hb}$ only by its large size, to explore a molecular size-dependent protection of vascular NO signaling in cerebral arteries. NO-mediated relaxation of basilar artery segments was completely interrupted by $\mathrm{Hb}$, but it remained intact in the presence of equal concentrations of the Hb-haptoglobin complex or polymerized $\mathrm{Hb}$ (Figure $5 \mathrm{C}$ ). The differential effects of the 3 hemoproteins on vascular responses were mirrored by the divergent ability of the proteins to translocate from the subarachnoid CSF space into the brain parenchyma (Figure 5D). For these mechanistic studies we injected trans-cyclooctene-labeled (TCO-labeled) Hb, Hb-haptoglobin, or polymerized $\mathrm{Hb}$ into the subarachnoid space of mice. TCO is a small tag, which can be visualized with a high signal-to-noise ratio on vibratome sections after a click-chemistry reaction with Cy5-conjugated tetrazine. Figure 5D shows that the small cellfree $\mathrm{Hb}$ delocalizes within 2 hours from the subarachnoid space deep into the mouse brain. This is in contrast to the Hb-haptoglobin complex and the polymerized $\mathrm{Hb}$, which remained completely excluded from the brain parenchyma. In the brains of the $\mathrm{Hb}$-haptoglobin and polymerized $\mathrm{Hb}$-injected animals positive fluorescence signals were only detected in the region of the injection trajectory. Figure 5E shows SEC-HPLC elution profiles of the 3 hemoproteins demonstrating the small size of $\mathrm{Hb}$, and the large size of $\mathrm{Hb}$-haptoglobin complexes and polymerized $\mathrm{Hb}$, respectively. From these studies, we hypothesized that size-restricted barriers may limit translocation of the Hb-haptoglobin complex from the subarachnoid CSF space into NO-sensitive compartments of the cerebral vasculature and the brain.

Haptoglobin blocks translocation of cell-free Hb from CSF to brain parenchyma and blood vessels in sheep. In a next experimental step, 
A
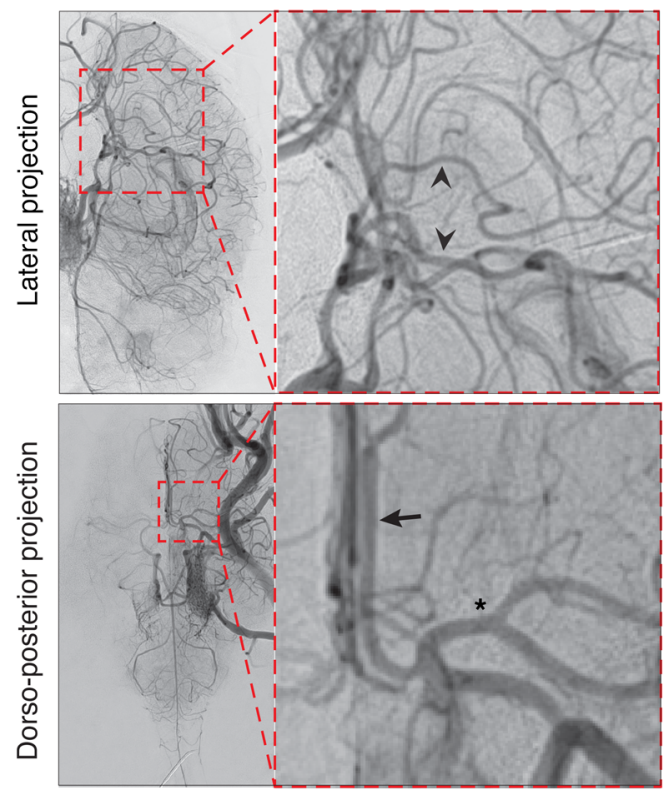

B

Baseline $60 \mathrm{~min}$

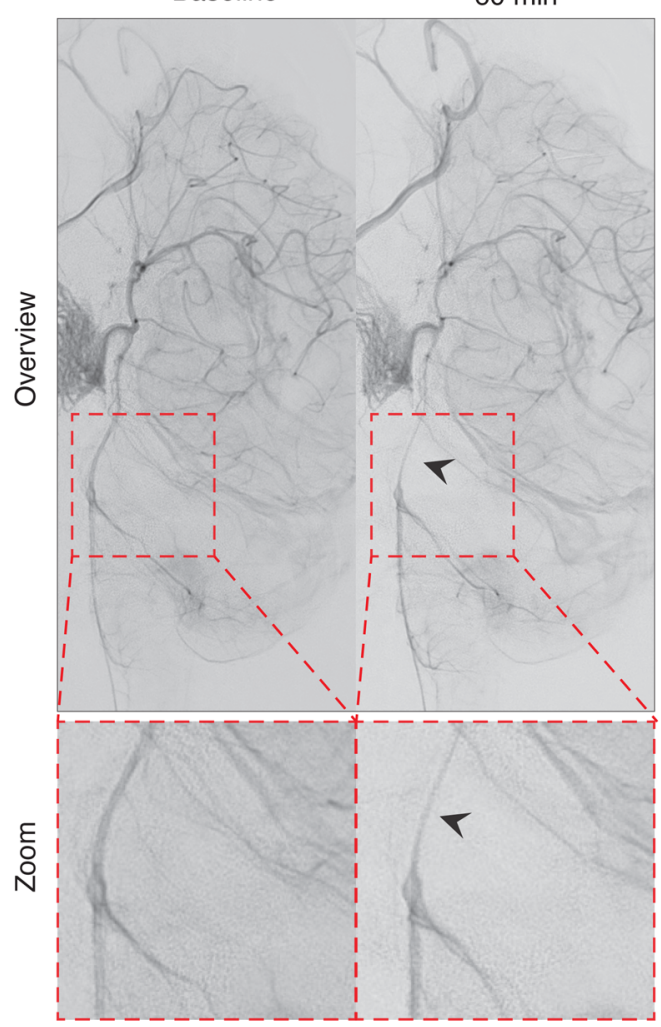

60 min after $\mathrm{Hb}$ infusion
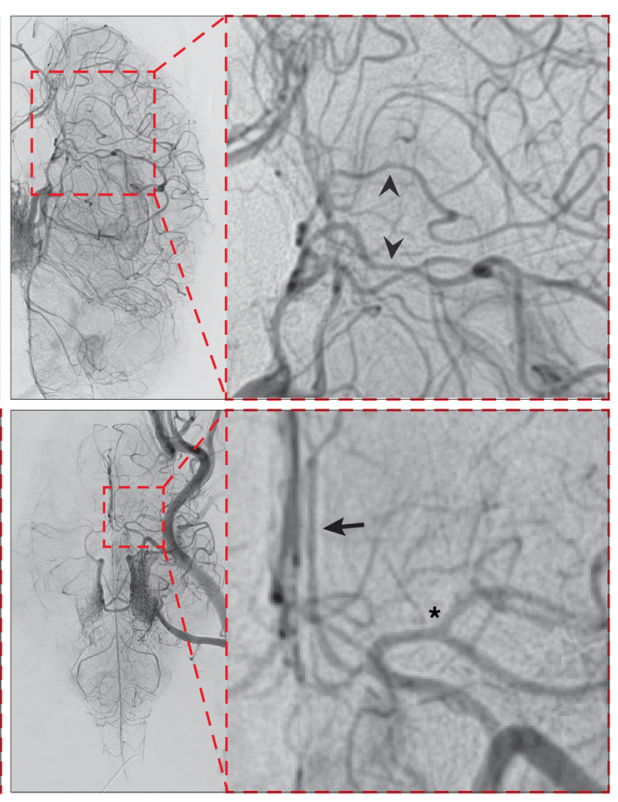

$\mathrm{Hb}-\mathrm{Hp}$
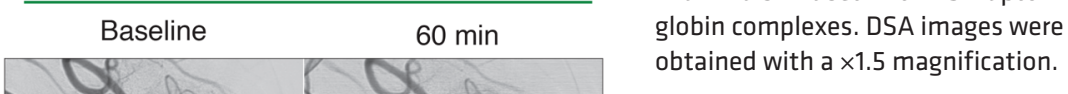

Figure 4. Hb-induced vasospasm after ventricular infusion in sheep.

(A) Angiograms illustrating segmental vasospasms in major vascular territories, including the middle cerebral artery in the lateral projection (arrowheads, upper panel), the anterior cerebral artery (arrow, lower panel), and the middle cerebral artery (asterisk, lower panel) in the dorsoventral projection, 60 minutes after $\mathrm{Hb}$ infusion. The demonstrated angiograms are from the same experiment shown in Figure 3B. (Identical images are shown in Figure $3 \mathrm{~B}$ and Figure $4 \mathrm{~A}$ for the dorso-posterior projections at baseline). (B) Comparison of illustrative digital subtraction angiography in lateral projection of 2 sheep 60 minutes after infusion of $\mathrm{Hb}$ (left) or Hb-haptoglobin complexes (right). Segmental vasospasms of the basilar artery (arrow) were apparent 60 minutes after infusion of $\mathrm{Hb}$, whereas no segmental vasospasms could be detected in animals infused with $\mathrm{Hb}$-haptoglobin complexes. DSA images were we infused TCO-tagged $\mathrm{Hb}$ or $\mathrm{Hb}$-haptoglobin complexes into the CSF of sheep to further analyze distribution and translocation patterns of $\mathrm{Hb}$ in the brain and the cerebral vasculature. The fluorescence scans of $120-\mu \mathrm{m}$ sheep brain sections at 2 different sites of the forebrain and midbrain demonstrated that within 2 hours after infusion, the cell-free $\mathrm{Hb}$ was delocalized from the internal and external CSF spaces into the brain parenchyma, appearing as a rim of fluorescence signal along the internal and external CSF- brain interfaces (Figure 6A, left). This pattern of delocalization was absent in sheep brain sections that were infused with TCOHb-haptoglobin complexes (Figure 6A, right). For the illustration of the $\mathrm{Hb}$-haptoglobin complex distribution in Figure 6A, we chose a section of the forebrain where the tip of the ventricular catheter was placed slightly into the brain parenchyma. At this location, a small quantity of TCO-Hb-haptoglobin was directly injected into the brain tissue, serving as a positive control for the staining and 
A

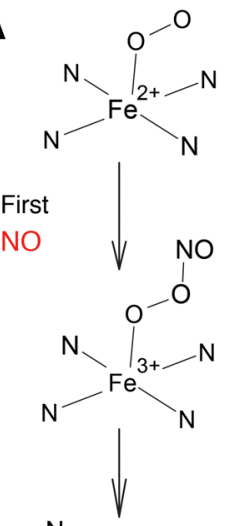<smiles>N[P+](N)(N)N[Nb](=O)(=O)O</smiles>

Second NO<smiles></smiles><smiles>NC(N)(O)[P+](N)(N)N</smiles><smiles>N=CN=N[P+](N)(N)N</smiles>

B

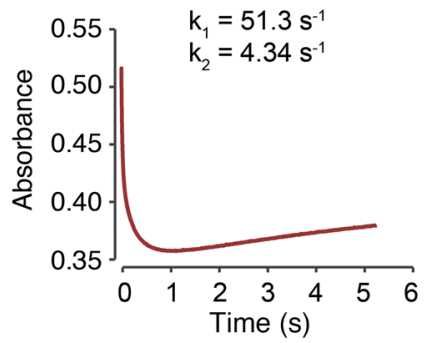

C

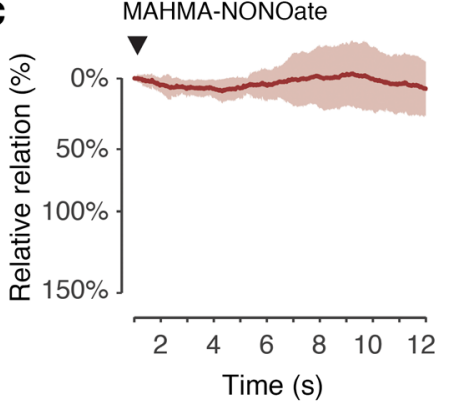

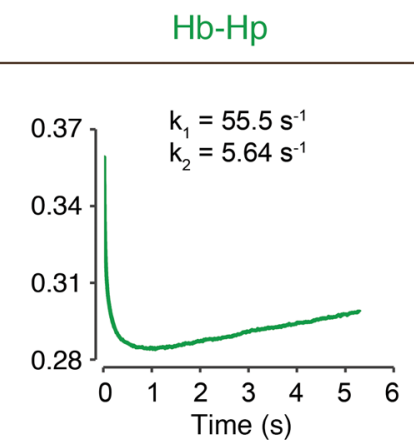

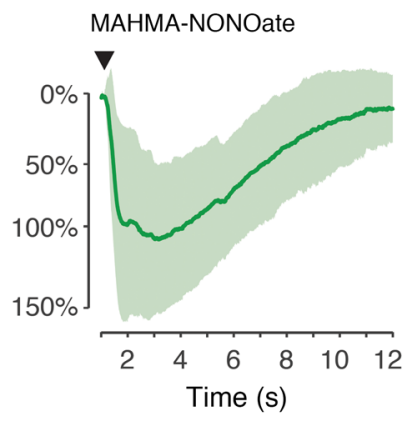

poly-Hb

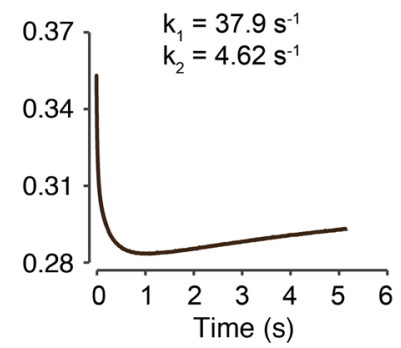

MAHMA-NONOate

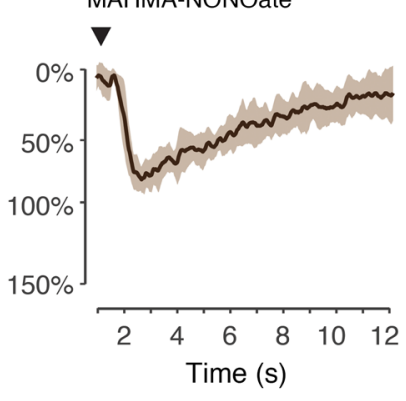

\section{D}

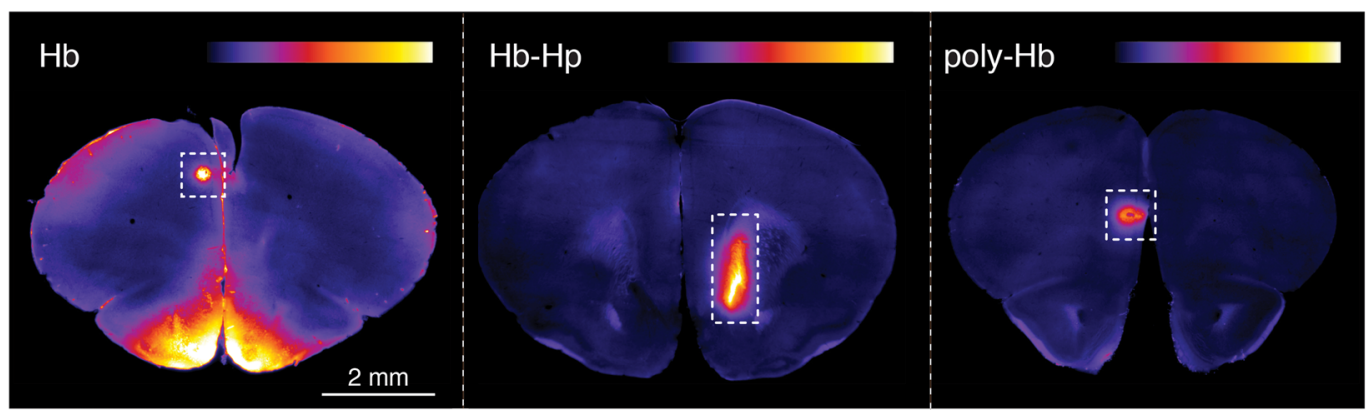

E

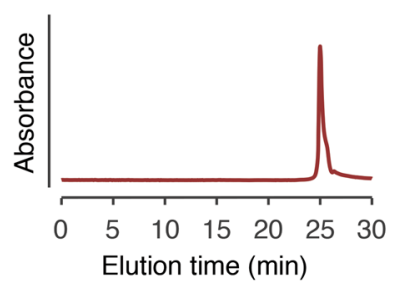

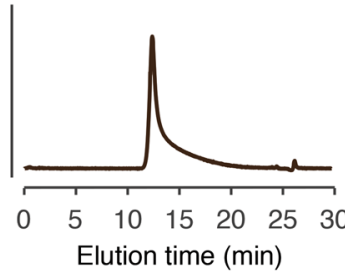

Figure 5. The protective function of $\mathrm{Hb}$-haptoglobin in the brain is determined by its molecular size. (A) Reaction sequence of oxyHb with $\mathrm{NO}$ under conditions of excess NO. In a 3-step reaction, oxyHb can scavenge up to 3 molecules of NO. (B) NO-reaction kinetics of Hb (left), Hb-haptoglobin (middle), or polymerized $\mathrm{Hb}$ (right). Shown are absorbance changes at $405 \mathrm{~nm}$ after rapid mixing of $\mathrm{NO}$ and oxyHb in a stopped-flow spectrophotometer. Estimates of the reaction rates ( $\mathrm{k} 1$ and $\mathrm{k} 2$ ) were calculated by approximating the data with an equation defined by the sum of 3 exponential functions. (C) Tension traces (mean \pm SD) of porcine basilar arteries immersed in buffer containing $10 \mu \mathrm{M} \mathrm{Hb}$ (left; red, $n=16$ ), Hb-haptoglobin (middle; green, $n=16$ ), or polymerized $\mathrm{Hb}$ (right, brown, $n=8$ ). A bolus of MAHMA-NONOate was added (arrowhead) to induce NO-mediated dilation. (D) Coronal vibratome sections ( 2 mm anterior to the bregma) of mouse brains 2 hours after subarachnoid injection of TCO-labeled Hb (left), Hb-haptoglobin (middle) and polymerized Hb (right). The false-colored images represent the signal intensity of the injected compound after postmortem coupling to a fluorophore (tetrazine-5-TAMRA). Intraparenchymal delocalization is only observed in the mouse injected with $\mathrm{Hb}$ and not after injection of $\mathrm{Hb}$-haptoglobin and polymerized $\mathrm{Hb}$ (images are representative for $n=3$ per group). The signal in the area of the puncture channel (dashed boxes) serves as a positive control for the injection and labeling procedure. (E) SEC elution profiles of pure $\mathrm{Hb}$ (left; red), Hb-haptoglobin (middle; green), and polymerized Hb (right; brown) measured at $414 \mathrm{~nm}$ illustrate the different molecular size of the injected compounds.

imaging procedure. In addition, the only distinct Hb-haptoglobin signal could be recognized alongside small arteries penetrating from the pial surfaces into the brain. Before sacrificing the sheep, we collected CSF from the subarachnoid space for SEC and SDS-
PAGE analysis. Despite the absence of an Hb-haptoglobin signal in brain sections, we identified the large Hb-haptoglobin complex by SEC and SDS-PAGE in the CSF, which was sampled at the end of the experiment before sacrificing the animal (Figure 6, B and 

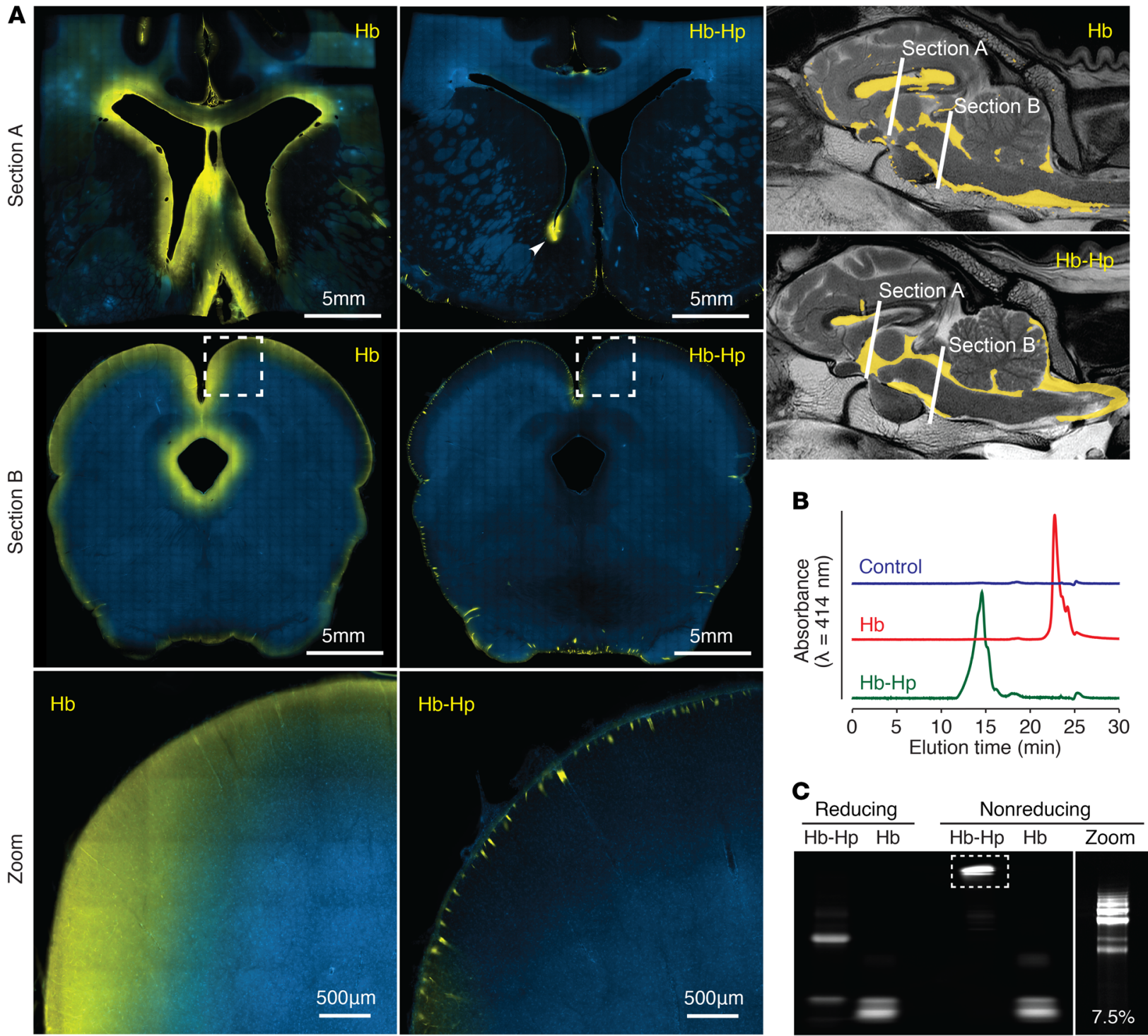

Figure 6. Haptoglobin blocks translocalization of $\mathrm{Hb}$ from CSF into the brain parenchyma. (A) Representative fluorescent images of sheep brain sections $(120 \mu \mathrm{m})$ after injection of TCO-labeled Hb (left panels) and Hb-haptoglobin complexes (right panels), stained for nuclei (blue) and the labeled compound (tetrazine-Cy5, yellow). Hb translocates from the ventricular system through the ependymal barrier and from the subarachnoid space through the glia limitans into the brain interstitial space (images are representative for $n=4$ animals). This delocalization cannot be observed when $\mathrm{Hb}$ is bound to haptoglobin (images are representative for $n=5$ animals). Fluorescent $\mathrm{Hb}$-haptoglobin complexes can be recognized only along penetrating cortical vessels. In Section A, a small amount of $\mathrm{Hb}$-haptoglobin complexes was injected directly into the brain parenchyma at the tip of the external ventricular drain catheter, serving as a positive control for the staining and imaging procedure (arrow head). Whole-slide scans were produced by stitching single images obtained at $\times 10$ magnification. Scale bars are indicated in the figure. (B) Before animals were sacrificed, CSF samples from Hb-and Hb-haptoglobin-treated sheep were collected from the subarachnoid space and analyzed by SEC-HPLC, indicating typical elution profiles of cell-free Hb and large Hb-haptoglobin complexes. CSF sample of a sheep before infusion (blue trace), CSF sample of a sheep infused with Hb (red trace), CSF sample of a sheep infused with $\mathrm{Hb}$-haptoglobin complexes (green trace). (C) The same CSF samples were reacted ex vivo with Cy5 tetrazine and analyzed by SDS page. The bands represent $\mathrm{a} / \mathrm{b}$ globin chains in cell-free $\mathrm{Hb}(\mathrm{Hb})$ and $\mathrm{Hb}$-haptoglobin complex $(\mathrm{Hb}-\mathrm{Hp})$. Under nonreducing conditions, the specific ladder pattern of the different type 2-2 haptoglobin polymers is clearly visible when analyzed on a gel optimized for high resolution of large proteins (7.5\%).

C). Collectively, these data support that cell-free $\mathrm{Hb}$ translocates from the CSF to the brain parenchyma and that the Hb-haptoglobin complex remains compartmentalized in the CSF space.

In a next series of imaging studies, we evaluated whether cellfree $\mathrm{Hb}$ could translocate from the CSF into the vascular smooth muscle cell layer of cerebral arteries, where NO mediates its vasodilatory signal. This translocation may be a key pathophysiological pathway for cell-free $\mathrm{Hb}$ acting as a cerebral artery vasoconstric- tor, which could be blocked for the large Hb-haptoglobin complex. For examination of vascular structures by confocal microscopy, sheep brain sections from animals infused with TCO-Hb or TCO$\mathrm{Hb}$-haptoglobin were stained for vascular smooth muscle cells and for the aquaporin-4-positive astrocyte end-feet, and confocal micrographs of several small arteries of different calibers in the periventricular area of the midbrain were taken. Cell-free $\mathrm{Hb}$ delocalized across the astrocyte barrier into the brain tissue and 
additionally into the vascular smooth muscle layer, reaching the subendothelial space (Figure 7). By contrast, the Hb-haptoglobin complex remained compartmentalized at a high concentration within the CSF-filled perivascular space (Virchow-Robin space) of penetrating arteries. This space is delineated by the outermost layer of the arteries (i.e., the adventitia) on one side and by the astrocyte end-feet on the other side (i.e., the glia limitans). These observations likely explain why cell-free $\mathrm{Hb}$ but not the large $\mathrm{Hb}$-haptoglobin complex interrupts vasodilatory NO signaling in cerebral arteries and thereby induces vasospasm.

To link the observed perivascular $\mathrm{Hb}$ exposure of small parenchymal vessels (diameter range of $50-100 \mu \mathrm{m}$ ) to the functional readouts for large cerebral arteries, we performed a histomorphometric analysis. For this purpose, we stained $120-\mu \mathrm{m}$ sheep brain sections through the fourth ventricle for smooth muscle cells (Supplemental Figure 5A). Confocal images of the small alpha smooth muscle actin-positive ( $\alpha$ SMA-positive) vessels in the tela choroidea were acquired by a researcher blinded to the treatments ( $n=3$ sheep per group, a total of 25 images of Hb-treated sheep, 32 images for Hb-haptoglobin-treated sheep). We have chosen the periventricular area for this analysis because this tissue is very rapidly exposed to the intraventricularly instilled fixative, ensuring excellent structural preservation after euthanasia. For each vessel, the lumen and total cross-sectional areas were quantified based on the inner and outer circumference of the $\alpha \mathrm{SMA}$-positive structures, which were manually determined by 3 blinded researchers (Supplemental Figure 5B). While vessels with a contracted appearance were abundant in all $\mathrm{Hb}$-infused animals, none could be found in Hb-haptoglobin-infused animals (Supplemental Figure 5C). Supplemental Figure 5, D and E, shows the quantitative analysis of the luminal fraction areas of all analyzed vessels, as well as the mean per sheep. In both ways of analysis, the differences were statistically significant with smaller fractional lumen areas of $\mathrm{Hb}$ compared with Hb-haptoglobin-treated animals.

\section{Discussion}

In patients with aSAH, erythrolysis in the subarachnoid space leads to an accumulation of cell-free $\mathrm{Hb}$ exceeding haptoglobin scavenger capacity in the CSF. We confirmed that this unbound $\mathrm{Hb}$ is the principal dysregulator of vascular NO signaling, and thus promotes spasms in cerebral arteries and smaller parenchymal arterioles. Our results show that delocalization of cell-free $\mathrm{Hb}$ from CSF into vascular structures is the primary cause of this pathophysiology, which can be prevented by size-dependent compartmentalization of $\mathrm{Hb}$ in the CSF by the formation of large $\mathrm{Hb}$-haptoglobin complexes. We further identified delocalization of $\mathrm{Hb}$ into the brain parenchyma as another pathway of Hb-induced pathophysiology, which is also blocked by haptoglobin. These observations suggest that haptoglobin may control a spectrum of oxidative and $\mathrm{NO}-$ related toxicities that have been associated with cell-free $\mathrm{Hb}$, heme, and iron exposures within the brain (Figure 8).

We found no NO-depleting or vasoconstrictive activity in human CSF samples collected from patients shortly (1 or 2 days) after the inciting bleeding event. In contrast, samples collected at later phases of the disease displayed an erythrolysed protein signature that disrupted NO signaling and shifted vascular tone balance toward vasoconstriction. Selective removal of $\mathrm{Hb}$ from patient-derived CSF by a haptoglobin column was sufficient to abate this activity. Furthermore, the collective experimental data presented here suggest that a therapeutic intervention targeting compartmentalization of $\mathrm{Hb}$ within the CSF might be sufficient to prevent cerebral vasospasm. The slow time course of $\mathrm{Hb}$ accumulation in CSF after the inciting bleeding event provides a window of opportunity to restore the $\mathrm{Hb}$ binding capacity of CSF by exogenous haptoglobin.

The protective physiological effect of haptoglobin during systemic hemolysis has been related to the large molecular size of the Hb-haptoglobin complex $(15,43)$. The complex is too large to be filtered by the kidney or to extravasate from blood into the wall structures of coronary arteries or the interstitial space of the myocardium $(15,48)$. These observations helped to resolve the enigma that the biochemical reactivity, which was recognized as the source of cell-free $\mathrm{Hb}$ toxicity, was not substantially different in the case of the Hb-haptoglobin complex compared with unbound $\mathrm{Hb}$. According to the compartmentalization hypothesis, the potentially detrimental peroxidase and dioxygenase reactions of $\mathrm{Hb}$ remain uneventful as long as they occur within the circulatory plasma compartment. Here, we could extend and reinforce this hypothesis in experiments mimicking intracranial conditions, which are fundamentally different from the models of intravascular hemolysis that we have studied before.

In patient and sheep CSF, the reaction kinetics of cell-free $\mathrm{Hb}$ and the Hb-haptoglobin complexes with NO were similar, even though we adapted our experimental conditions to model the full 3-reaction kinetics of $\mathrm{Hb}$ with $\mathrm{NO}$ depicted in Figure 5B and Supplemental Figure 4. However, we discovered that haptoglobin was able to block 2 divergent pathways of cellfree $\mathrm{Hb}$ delocalization from CSF. First, haptoglobin prevented translocation of cell-free $\mathrm{Hb}$ from the CSF into the smooth muscle layer and subendothelial space of arterial vessels. We assume that the size-restricted interruption of this delocalization path by haptoglobin spatially separates the Hb-haptoglobin from functional $\mathrm{NO}$ and thus preserves vascular NO signaling, preventing vasospasms in small and large cerebral arteries. The finding that chemically crosslinked Hb-polymers - with a large molecular size but unaltered NO reactivity compared with $\mathrm{Hb}$ - not only mimicked the spatial compartmentalization effect of the Hb-haptoglobin complex, but also showed the same NO-sparing effect in ex vivo vascular function studies, supported this assumption. Second, haptoglobin prevented translocation of cell-free $\mathrm{Hb}$ through the inner and outer CSFbrain barrier into the brain interstitial space (i.e., through the ependymal layer and glia limitans) $(49,50)$. Again, we found no evidence that the Hb-haptoglobin complex could cross this anatomical barrier, which is permeable to proteins up to $70 \mathrm{kDa}$ $(49,50)$. Prevention of $\mathrm{Hb}$ delocalization into the brain's interstitial space may avert $\mathrm{Hb}$-triggered oxidative stress to neural tissue $(43,51)$ and spare NO signaling within the neurovascular unit. Furthermore, prevention of this delocalization may reduce Hb-triggered microthrombosis (34-36), neuroinflammation $(39,40)$, and pathological electrical activity (i.e., cortical spreading depression; CSD) (37).

Vasodilatory NO signaling can be disrupted at several levels after aSAH $(41,52)$. Our studies focused on the sink theory, by 
$\mathrm{Hb}$

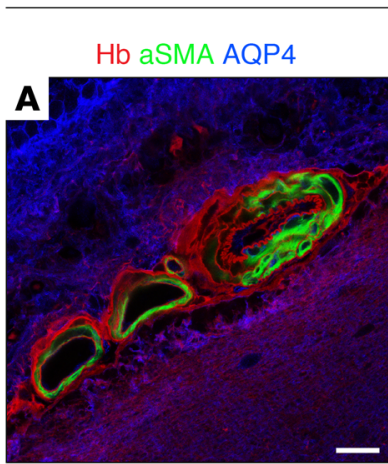

B

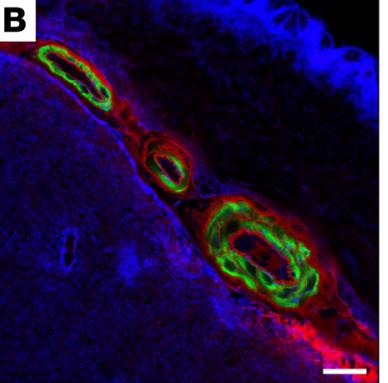

c

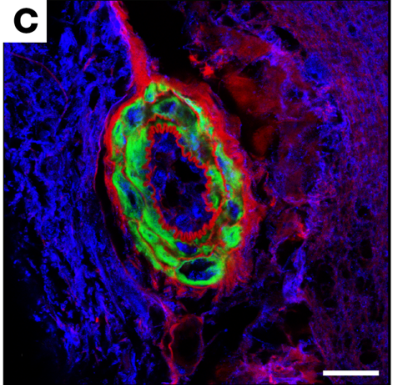

D
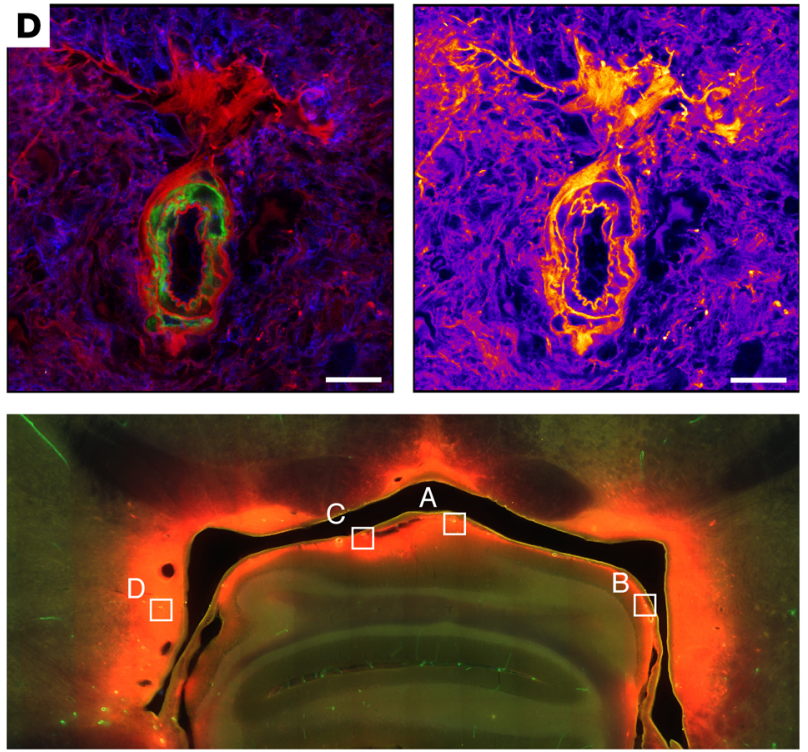

$\mathrm{Hb}-\mathrm{Hp}$

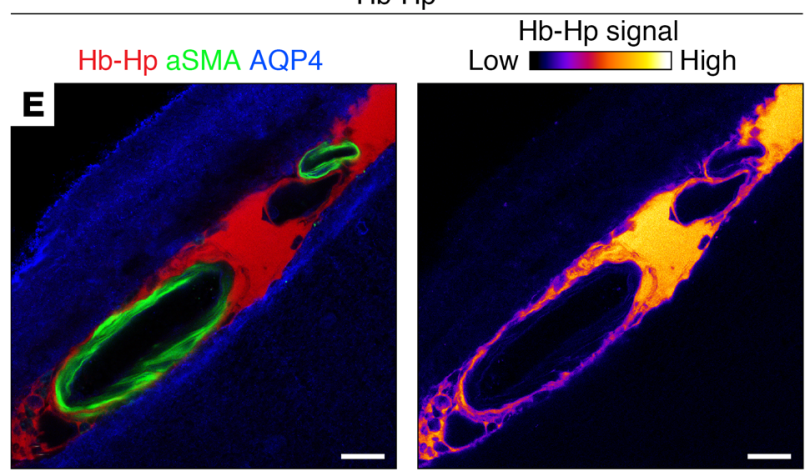

$\mathbf{F}$
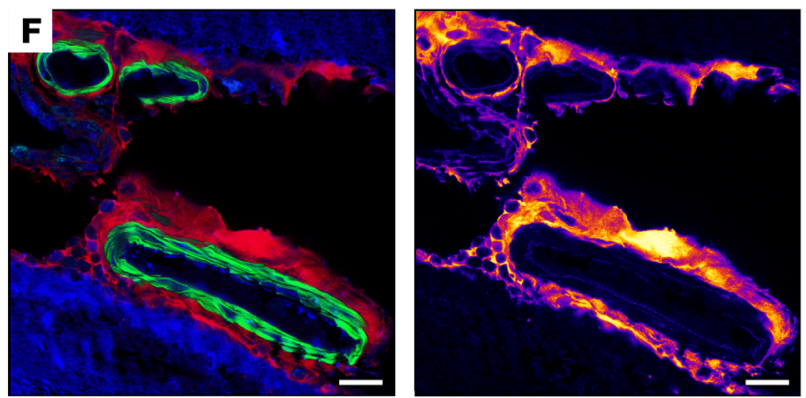

G
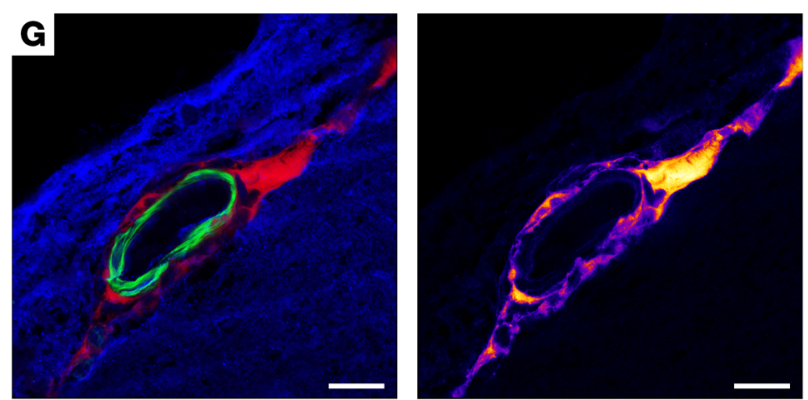

$\mathbf{H}$
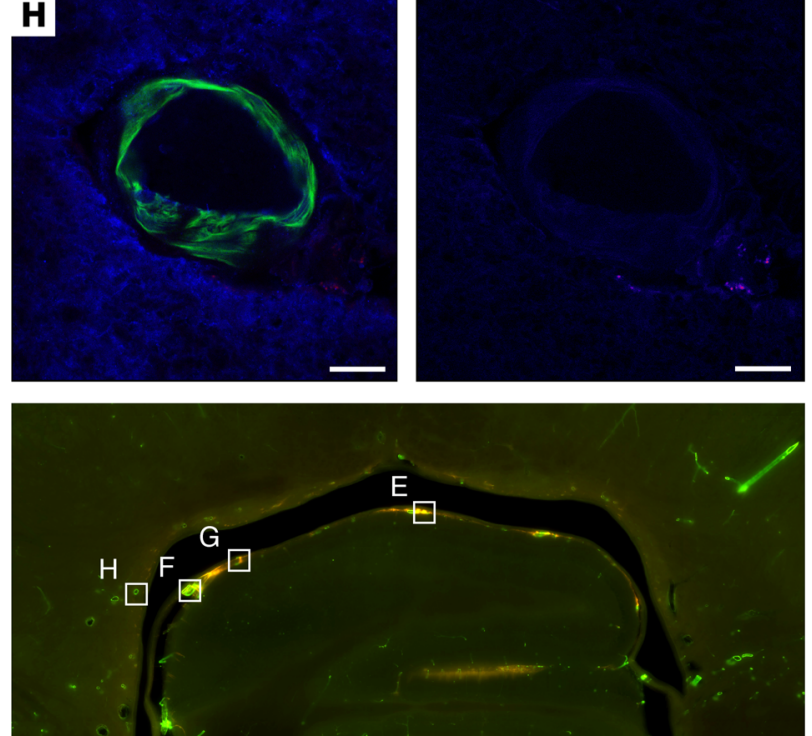

Figure 7. Haptoglobin blocks translocation of $\mathbf{H b}$ from CSF into the brain vasculature. Representative confocal images of small arteries in the periventricular area of the midbrain from a sheep after infusion of TCO-labeled Hb (A-D) or Hb-haptoglobin complexes (E-H). The 120- $\mu \mathrm{m}$ vibratome sections were immunostained for vascular smooth muscle cells ( $\alpha$ SMA, green), astrocyte end-feet (AQP4, blue), and TCO-labeled Hb (tetrazine-Cy5, red). False-colored images (Hb-signal) display only the signal of the labeled hemoprotein from the corresponding image. The delocalization of cell-free $\mathrm{Hb}$ from the CSF into arterial vessel walls (smooth muscle cell layer) and the brain parenchyma (astrocyte covered area) is blocked by haptoglobin (images are representative for $n=4$ animals injected with TCO-Hb, and $n=5$ animals injected with TCO-Hb-haptoglobin complex). Scale bars: $20 \mu \mathrm{m}$. Bottom images show a whole slide scan as an overview at $\times 10$ magnification. Boxes indicate the position of the vessels $(\mathbf{A}-\mathbf{H})$ that are shown with higher magnification above. 


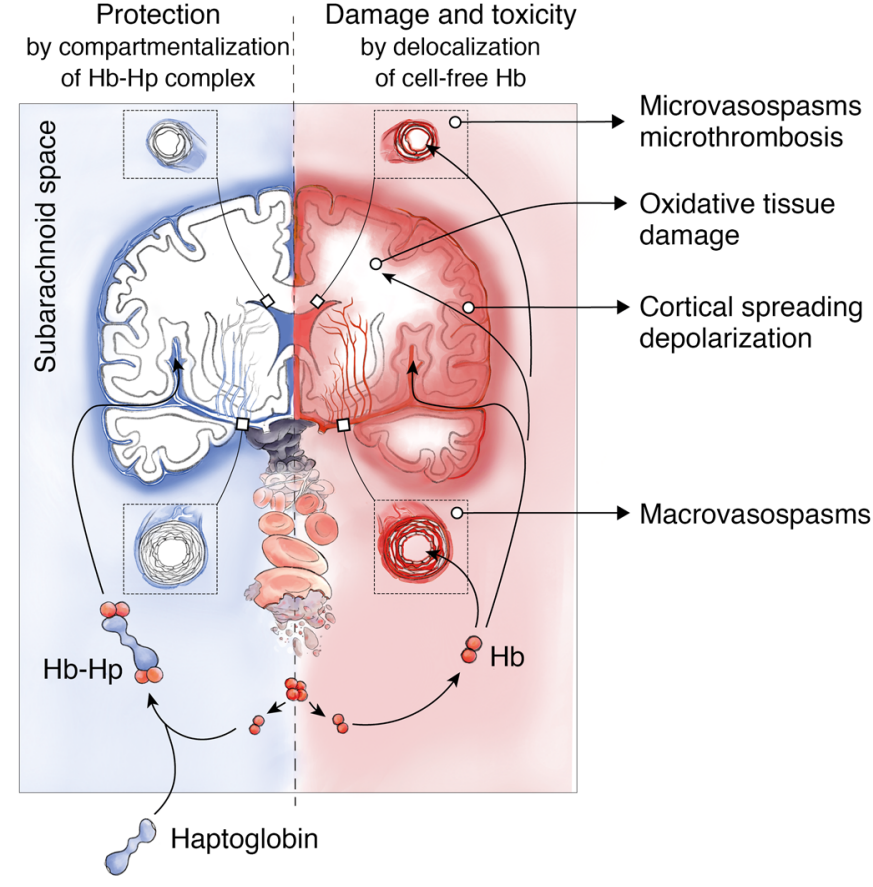

Figure 8. Mechanism of cell-free $\mathrm{Hb}$ toxicity and protection by complex formation of $\mathrm{Hb}$ with haptoglobin. Cell-free $\mathrm{Hb}$ is released from lysing erythrocytes in the subarachnoid blood clot after aSAH and distributes within the CSF (red). Delocalization of small Hb dimers ( $32 \mathrm{kDa}$ ) into vessel walls of resistance arteries causes macro- and microvascular vasospasms through NO depletion in the vascular wall. Delocalization into the brain parenchyma may cause oxidative damage and alter the interstitial microenvironment. Therapeutic haptoglobin injected into the ventricular system binds $\mathrm{Hb}$ in the subarachnoid space and prevents delocalization of the large $\mathrm{Hb}$-haptoglobin complex (>150 kDa), thereby attenuating toxicity (blue).

which oxyHb reacts with $\mathrm{NO}$ and depletes the vasodilator within the vascular wall or in neuronal tissues. Several other mechanisms of NO dysfunction have also been demonstrated; however, the quantitative contribution toward pathophysiology continues to be defined. Perivascular nerve fibers expressing nitric oxide synthase (NOS) among other vasoactive substances are critical for the regulation of cerebral arterial vascular tone $(53,54)$. These NOS-expressing neurons disappear after aSAH, presumably as a result of $\mathrm{Hb}$ and heme toxicity $(55,56)$. It has also been observed that the endogenous NOS inhibitor asymmetric dimethyl arginine (ADMA) accumulates in CSF after aSAH (57).

Additionally, $\mathrm{Hb}$, heme, and heme-degradation products can exert vascular effects via NO-independent mechanisms. Oxidation of bilirubin, the ultimate heme-metabolism product, results in the accumulation of bilirubin oxidation products (BOXes) and propentdyopents in patient CSF (58-60). Heme, BOXes, and propentdyopents can all promote vasoconstriction by acting on large conductance $\mathrm{BK}_{\mathrm{Ca}}$ potassium channels in astrocytes and vascular smooth muscle cells (60-62). Finally, oxyHb can stimulate production of the potent vasoconstrictor, endothelin $(63,64)$, and alter expression of calcium and potassium channels in cerebral arteries (65-67). Cell-free $\mathrm{Hb}$ and heme may also accelerate acute and chronic inflammatory processes promoting more chronic disruption of vascular homeostasis, which occurs alongside the NO depletion-related vasoconstriction after aSAH (68).
In relation to the NO-sink framework, the protective mechanism underlying our observations is related to the large molecular size of the Hb-haptoglobin complex restricting access of cell-free $\mathrm{Hb}$ to NO-sensitive structures in the vascular wall. Haptoglobin demonstrates additional protective functions, which are related to its structural stabilization of bound $\mathrm{Hb}$ molecule dimers. We have shown that free radicals are stabilized and less reactive in the Hb-haptoglobin complex compared with free $\mathrm{Hb}(69,70)$, that the complex has an enhanced resistance against oxidative damage $(71,72)$, and that the oxidant heme cannot be released and transferred from $\mathrm{Hb}-$ haptoglobin to lipid compartments (73). In many experimental models, these functions of haptoglobin translate into antioxidant and cytoprotective effects $(44,74,75)$. It remains to be investigated how these additional functions of haptoglobin may protect against a broader range of $\mathrm{Hb}$ - and heme-driven pathologies after aSAH.

We noted several limitations of our study that need to be addressed before an haptoglobin-based therapy can be further developed for a clinical trial in patients with aSAH. First, our studies were designed to dissect the toxicity of cell-free $\mathrm{Hb}$ in CSF as a distinct and targetable pathophysiological factor potentially accelerating the development of secondary neurological damage after aSAH. Our models do not account for mechanisms of early brain injury (EBI) caused by aneurysm rupture in aSAH patients (41, 76). Interspecies differences and mode of bleeding induction are known to limit translation of animal model data in SAH research to clinical practice (77). We have tried to compensate for this limitation by the use of different species and the use of CSF samples of aSAH patients in ex vivo vascular function experiments.

Various haptoglobin phenotypes occur in humans. Haptoglobin 1-1 is a homodimeric molecule with a molecular size of 84 $\mathrm{kDa}$ without bound $\mathrm{Hb}$ and $148 \mathrm{kDa}$ with $2 \mathrm{Hb}$ dimers bound at each pole of the protein (42). Haptoglobin 2-2 and haptoglobin 2-1 are heterogeneous mixtures of larger polymers. Earlier hypotheses that outcomes of aSAH depended on haptoglobin phenotypes have been refuted (78), and our own studies could not reveal significant biochemical or physiological differences between the binding and detoxifying effects of haptoglobin 1-1 and haptoglobin 2-2 in vitro or in the systemic circulation of guinea pigs (79). In this study, we used a plasma-derived haptoglobin product, which is almost exclusively composed of larger haptoglobin polymers (haptoglobin 2-2 and haptoglobin 2-1). Therefore, we cannot make predictions regarding the functionality of haptoglobin 1-1 to detoxify cell-free $\mathrm{Hb}$ in the CSF. A homodimeric product, plasmaderived or recombinant, may be advantageous as a therapeutic compound compared with the heterogenous mixture of Type 2-2 polymers because it could be better characterized and may be formulated at a higher concentration with less aggregation and viscosity. These features may prove to be a critical advantage for a tightly volume-restricted intrathecal administration. However, since compartmentalization due to large molecular size proved to be the definite mechanism of protection, a careful comparison of the protective effect of the smaller haptoglobin 1-1 versus the larger haptoglobin 2-2 needs to be carried out.

Another open question that needs to be addressed relates to the clearance of Hb-haptoglobin complex from the CSF. We assume that a large proportion of the Hb-haptoglobin complexes formed in vivo could be removed by CSF drainage. However, 
we still expect that some Hb-haptoglobin complexes would be retained in more secluded areas of the subarachnoid space, such as within the widely ramified Virchow-Robin spaces. Macrophages clear Hb-haptoglobin complexes by the CD163 scavenger receptor, and have a high capacity to metabolize heme by heme-oxygenase $(80,81)$. However, few studies have addressed this clearance pathway in the CSF compartment (47), and it will be critical to further elucidate its efficiency after therapeutic haptoglobin supplementation. Additionally, it will be essential to understand how Hb-haptoglobin clearance may modulate macrophage phenotypes and inflammatory functions.

The last question relates to the technical feasibility of our concept. The typical bleeding volume in aSAH is $35 \mathrm{~mL}$ (82), which contains 4-5 g of cell-free $\mathrm{Hb}$. We expect that an optimized therapeutic formula should contain at least $100 \mathrm{mg} / \mathrm{mL}$ of active haptoglobin, and $40-50 \mathrm{~mL}$ of such a therapeutic formula should be administered into the CSF of a patient during a treatment course if all $\mathrm{Hb}$ in the clot should be captured. Although this volume seems large, the slow erythrolysis over several days provides a significant time window for a fractionated dosing schedule. Furthermore, the HPLC and wire-myography methods that we describe in this paper may support personalized dosing schemes based on functional target effects. Finally, we do not know how haptoglobin may be distributed within the subarachnoid space when a subarachnoid blood clot compromises physiologic flow and free diffusion in the CSF. Mechanical obstruction would likely reduce the accessibility of cell-free $\mathrm{Hb}$ within the clot and in the vicinity of the culprit blood vessel (83). However, as demonstrated in our patients and in the sheep model, cell-free $\mathrm{Hb}$ distributes in the CSF after erythrolysis, well beyond the site of the initial bleeding with potentially far-reaching toxic effects, and we consider this fraction of $\mathrm{Hb}$ as the accessible target of a haptoglobin-based therapeutic formula.

Future treatment strategies to improve neurological outcomes after aSAH will likely leverage the synergistic effects of multiple drugs. Currently, the calcium channel blocker nimodipine is the only drug approved for DIND prevention in the United States and Europe $(84,85)$. Despite ambiguous scientific evidence, intraarterial mechanical or chemical angioplasty is generally accepted as rescue therapy for selected patients with DIND and refractory cerebral vasospasms $(84,85)$. Endothelin receptor blockers reportedly reversed angiographic vasospasms in clinical trials but failed to improve neurological outcomes $(86,87)$, likely because of the significant systemic adverse effects. Additionally, therapeutic approaches limited to reversal of vasospasm in large arteries may not sufficiently address the cascading pathophysiology of DIND, which starts with the rupture of the aneurysm leading to early brain damage $(41,76,88)$, and which is subsequently amplified by secondary hits such as accumulation of cell-free $\mathrm{Hb}$ in CSF.

Based on the recognized importance of dysfunctional NO signaling in the pathophysiology of vasospasm and DIND, there remains a keen interest in the use of NO donors as preventive or rescue therapies. NO can be delivered by systemic administration of NO-releasing molecules such as sodium nitroprusside, nitroglycerin, molsidomine, or NONOates, by inhalation of NO gas, or locally, either via an arterial catheter during angiography or into the CSF (89-91). In mice, inhalation of NO reduced microvascular vasospasm, attenuated brain damage, and improved neurological outcome in an experimental model of SAH (92). Nitrite is another $\mathrm{NO}$ donor, which liberates $\mathrm{NO}$ in a reaction with deoxygenated $\mathrm{Hb}$ (93). Intravenous nitrite was effective in preventing and reversing cerebral artery vasospasm in a primate model of SAH, and demonstrated an acceptable side effect profile in humans $(33,94,95)$. Hypothetically, the local NO-sparing effect of haptoglobin may support the concomitant use of NO donors or other vasodilatory drugs, leveraging synergistic activities. Furthermore, the strong $\mathrm{Hb}$-stabilizing and antioxidant functions of $\mathrm{Hp}$ may alleviate prooxidant adverse effects of metHb formation that are associated with the use of NO donors $(73,96)$.

In summary, we defined $\mathrm{Hb}$ delocalization from CSF into cerebral vasculature and brain parenchyma as the most upstream mechanism of $\mathrm{Hb}$ toxicity after aSAH. Therapeutic haptoglobin supplementation compartmentalizes $\mathrm{Hb}$ in CSF and restores the physiological NO signaling in cerebral vasculature, which shifts the balance toward vasodilation. We conclude that intraventricular haptoglobin administration may present a useful therapeutic approach to reduce the toxicity of cell-free $\mathrm{Hb}$ in patients with hemorrhages into CSF.

\section{Methods}

Hemoglobin, haptoglobin, Hb-haptoglobin complexes, and polymerized hemoglobin. Hb was purified from sheep blood or expired human blood concentrates as previously described (97). Hb concentrations were determined by spectrophotometry as described and are given as molar concentrations of total heme $(1 \mathrm{M} \mathrm{Hb}$ tetramer is, therefore, equivalent to $4 \mathrm{M}$ heme) (73). For all $\mathrm{Hb}$ used in these studies, the fraction of ferrous oxyHb $\left(\mathrm{HbFe}^{2+} \mathrm{O}_{2}\right)$ was always greater than $98 \%$ as determined by spectrophotometry, unless stated otherwise. Haptoglobin from human plasma (phenotype 2-2 predominant) was obtained from CSL Behring. The identity, purity, and function of the protein were confirmed by liquid chromatography-tandem mass spectrometry (LC-MS/MS), PAGE, and SPR as previously described $(79,98) . \mathrm{Hb}$ binding capacity of the haptoglobin was quantified with SEC chromatography. After complex formation, the purity of the complex and the absence of free $\mathrm{Hb}$ was verified using SEC chromatography.

Polymerized bovine $\mathrm{Hb}$ was prepared at a 40:1 molar ratio of glutaraldehyde to human $\mathrm{Hb}$ as described (99). The preparation of $\mathrm{MnPP}-\mathrm{Hb}$ and MnPP-Hb-haptoglobin complexes is described in the supplement.

CSF samples from patients with aSAH. Patients with radiological high-grade (Fisher grade 3 or 4) aSAH and external ventricular drain insertion who were admitted to our Neurocritical Care Unit, University Hospital of Zurich (an academic tertiary care center), between April 2017 and December 2018 were included in a consecutive study approved by the local ethical review board of the canton of Zurich. Written consent was obtained from all patients or their legal representatives before study inclusion.

HPLC measurements. Samples containing $\mathrm{Hb}$ and/or Hb-haptoglobin were separated on an analytical BioSep-SEC-s3000 $(600 \times$ $7.8 \mathrm{~mm})$ LC column coupled with a BioSep-SEC-s3000 $(75 \times 7.8 \mathrm{~mm})$ Guard Column (Phenomenex). Details are provided in the supplement.

Proteomics of CSF samples. The proteomics workflow including sample preparation and LC-MS/MS analysis is described in the Supplemental Methods.

Measurement of $\mathrm{Hb}$-NO reaction by stopped-flow spectrophotometry. The $\mathrm{HbO}_{2}$ reaction with $\mathrm{NO}$ was measured with a SX18MV 
stopped-flow spectrophotometer (Applied Photophysics Ltd.) (100). Details of the measurement and data analysis are provided in the supplement.

Ex vivo vascular function experiments. Vascular function experiments were performed in a Multi-Channel Myograph System 620 M (Danish Myo Technology) as described in the supplement. The NO-mediated vasodilatory responses were induced by the addition of MAHMA-NONOate (ENZO Life Sciences). If not otherwise stated, the recorded $\mathrm{Hb}$-induced vascular function responses were normalized relative to maximum $\mathrm{NO}$ dilatation without $\mathrm{Hb}$ exposure (equal to $100 \%)$ and the level of tonic contraction before addition of MAHMANONOate (equal to $0 \%$ ), respectively, during a single experiment.

Sheep model. Animal studies were conducted according to the Swiss legal requirements for animal protection and welfare (TschG 455) and received ethical approval from the cantonal veterinary authorities "Kantonale Tierversuchskommission Zürich" (permission no. ZH234/17). Swiss alpine sheep, age 2-4 years, were obtained from the Staffelegghof.

Under general anesthesia, female ventilated sheep were instrumented with a left frontal external ventricular drain (DePuys Synthes) and a suboccipital spinal needle (Dalhausen) for CSF sampling from the subarachnoid space, and a right frontal multimodal neuromonitoring probe (Luciole Medicale AG). Illustration of the experimental setup is provided in Supplemental Figure 6. For controlled ventricular injections of aCSF, $\mathrm{Hb}$, haptoglobin, or Hb-haptoglobin complexes, a PHD Ultra syringe pump (Harvard Apparatus) was connected to the external ventricular drain. Ventricular injections were performed with maximal flow rates of $30 \mathrm{~mL} / \mathrm{h}$, and intracranial pressure maintained constant. A detailed description of the model is provided in the Supplemental Methods.

Digital subtraction angiography (DSA). Digital subtraction angiography was performed in an Allura Clarity angiography suite (Philips) with an angiographic $5 \mathrm{~F}$ catheter (Cordis) placed into the largest anastomosis between the right maxillary artery and the extradural rete mirabile. Details of the procedure, image processing, and data analysis are provided in the Supplemental Methods and in Supplemental Figure 3.

Magnetic resonance imaging. In vivo magnetic resonance imaging was performed in a clinical 3 Tesla MRI unit (Philips Ingenia). Details are provided in the supplement.

Mouse model. Wild-type male C57BL/6J mice (10-12 weeks, total $n=9$ ) were obtained from Charles River and maintained at the Laboratory Animal Services Center of the University of Zurich and treated in accordance with the guidelines of the Swiss Federal Veterinary Office. The studies were conducted according to the Swiss legal requirements for animal protection and welfare (TschG 455) and received ethical approval from the cantonal veterinary authorities "Kantonale Tierversuchskommission Zürich" (permission no. ZH073/17). Details of the stereotactic injection model and sample preparation are provided in the supplement.

Histology. Details of sample preparation and staining procedures including identity of antibodies are provided in the supplement.
Statistics. We used JMP software from SAS (versions 13 or 14) for all analyses. Data are presented with all data points plotted. Overlayed diamonds represent mean, 95\% CI, and overlap marks (horizontal lines above and below the mean line), which define statistical significant difference between groups if not overlapping $(P<0.05)$. Groups were compared with 1-way ANOVA, applying a Tukey-Kramer posttest correcting for multiple comparisons. A $P$ value of less than 0.05 was considered statistically significant.

Study approval. All animal studies were approved by the cantonal veterinary authorities "Kantonale Tierversuchskommission Zürich." The clinical study (CSF sampling) was approved by the local ethical review board of the Kanton of Zurich, and written consent was obtained from all patients or their legal representatives.

\section{Author contributions}

$\mathrm{MH}$ and RMB designed the study, performed experiments, analyzed data, and wrote the paper. CAS designed and performed experiments. HR and MD coordinated and performed animal studies. VA performed and analyzed angiography studies. KA performed animal studies, analyzed data, and wrote the paper. LM designed, performed, and analyzed stopped flow experiments. RV, JWD, PWK, and JHB performed experiments. FV wrote the paper. ZK performed and analyzed angiography studies. LR provided critical input and provided patient samples. AFP and ISP provided essential materials. $\mathrm{RH}$ analyzed data and generated illustrations. EK designed the clinical study and collected patient material. PWB provided essential material and wrote the paper. PRK coordinated large animal experiments. DJS conceptualized the project, designed the study, analyzed data, and wrote the paper. Authorship order for the 2 co-first authors was determined based on their time of entry into the project $(\mathrm{MH}$ entered the project before RMB).

\section{Acknowledgments}

The work was supported by Innosuisse (grant 19300.1 PF to DJS and $\mathrm{MH}$ ), the Swiss Heart Foundation (to DJS), and by the Swiss National Science Foundation and the Swiss Cancer Research Foundation (MD-PhD 4221-06-2017 to RMB). We thank Nadja Schulthess and Kerstin Hansen for technical support, and Reinhard Kissner for help with the stop-flow experiments. We greatly appreciate the generous support of the radiology service team from the Clinic for Diagnostic Imaging at the Tierspital Zürich. We thank the staff of the Functional Genomic Center Zurich (FGCZ) for support with the mass spectrometry analysis of CSF samples.

Address correspondence to: Dominik Schaer, Division of Internal Medicine, University of Zurich, Ramistrasse 100, CH-8091 Zurich, Switzerland. Phone: 41.44.255.1111; Email: dominik.schaer@usz.ch. Or to: Michael Hugelshofer, Department of Neurosurgery, University of Zurich, Frauenklinikstrasse 10, CH-8091 Zurich, Switzerland. Phone: 41.44.255.1111; Email: michael.hugelshofer@usz.ch.
1. Hughes JD, et al. Estimating the global incidence of aneurysmal subarachnoid hemorrhage: a systematic review for central nervous system vascular lesions and meta-analysis of ruptured aneurysms. World Neurosurg. 2018;115:430-447.e7.
2. Rincon F, Rossenwasser RH, Dumont A. The epidemiology of admissions of nontraumatic subarachnoid hemorrhage in the United States. Neurosurgery. 2013;73(2):217-223.

3. van Gijn J, Kerr RS, Rinkel GJE. Subarachnoid haemorrhage. Lancet. 2007;369(9558):306-318.

4. Loch Macdonald R. Delayed neurological deterioration after subarachnoid haemorrhage. Nat Rev Neurol. 2013;10(1):44-58.

5. Eagles ME, Tso MK, Macdonald RL. Cognitive 
impairment, functional outcome, and delayed cerebral ischemia after aneurysmal subarachnoid hemorrhage. World Neurosurg. 2019;124:e558e562.

6. Hugelshofer M, et al. Cell-free oxyhemoglobin in cerebrospinal fluid after aneurysmal subarachnoid hemorrhage: biomarker and potential therapeutic target. World Neurosurg. 2018;120:e660-e666.

7. Macdonald RL, Weir BK. A review of hemoglobin and the pathogenesis of cerebral vasospasm. Stroke. 1991;22(8):971-982.

8. Macdonald RL, Pluta RM, Zhang JH. Cerebral vasospasm after subarachnoid hemorrhage: the emerging revolution. Nat Clin Pract Neurol. 2007;3(5):256-263.

9. Macdonald RL, Weir BK, Grace MG, Martin TP, Doi M, Cook DA. Morphometric analysis of monkey cerebral arteries exposed in vivo to whole blood, oxyhemoglobin, methemoglobin, and bilirubin. Blood Vessels. 1991;28(6):498-510.

10. Reiter CD et al. Cell-free hemoglobin limits nitric oxide bioavailability in sickle-cell disease. $\mathrm{Nat}$ Med. 2002;8(12):1383-1389.

11. Baek JH et al. Hemoglobin-driven pathophysiology is an in vivo consequence of the red blood cell storage lesion that can be attenuated in guinea pigs by haptoglobin therapy. J Clin Invest . 2012;122(4):1444-1458.

12. Schaer DJ, Buehler PW, Alayash AI, Belcher JD, Vercellotti GM. Hemolysis and free hemoglobin revisited: exploring hemoglobin and hemin scavengers as a novel class of therapeutic proteins. Blood. 2013;121(8):1276-1284.

13. Donadee $\mathrm{C}$ et al. Nitric oxide scavenging by red blood cell microparticles and cell-free hemoglobin as a mechanism for the red cell storage lesion. Circulation. 2011;124(4):465-476.

14. Buehler PW, Alayash AI. Toxicities of hemoglobin solutions: in search of in vitro and in vivo model systems. Transfusion. 2004;44(10):1516-1530.

15. Schaer CA, et al. Haptoglobin preserves vascular nitric oxide signaling during hemolysis. Am J Respir Crit Care Med. 2016;193(10):1111-1122.

16. Alayash AI. Oxygen therapeutics: can we tame haemoglobin? Nat Rev Drug Discov. 2004;3(2):152-159.

17. Reeder BJ, Svistunenko DA, Cooper CE, Wilson MT. The radical and redox chemistry of myoglobin and hemoglobin: from in vitro studies to human pathology. Antioxid Redox Signal. 2004;6(6):954-966.

18. Doherty DH, et al. Rate of reaction with nitric oxide determines the hypertensive effect of cell-free hemoglobin. Nat Biotechnol. 1998;16(7):672-676.

19. Schechter AN, Gladwin MT. Hemoglobin and the paracrine and endocrine functions of nitric oxide. N EnglJ Med. 2003;348(15):1483-1485.

20. Gladwin MT, Kanias T, Kim-Shapiro DB. Hemolysis and cell-free hemoglobin drive an intrinsic mechanism for human disease. JClin Invest. 2012;122(4):1205-1208.

21. Garry PS, Ezra M, Rowland MJ, Westbrook J, Pattinson KT. The role of the nitric oxide pathway in brain injury and its treatment--from bench to bedside. Exp Neurol. 2015;263:235-243.

22. Esplugues JV. NO as a signalling molecule in the nervous system. Br JPharmacol. 2002;135(5):1079-1095.
23. Attwell D et al. Glial and neuronal control of brain blood flow. Nature. 2010;468(7321):232-243.

24. Katusic ZS, Austin SA. Endothelial nitric oxide: protector of a healthy mind. Eur Heart J. 2014;35(14):888-894.

25. Faraci FM, Brian JE. Nitric oxide and the cerebral circulation. Stroke. 1994;25(3):692-703.

26. Jeffers A, Gladwin MT, Kim-Shapiro DB. Computation of plasma hemoglobin nitric oxide scavenging in hemolytic anemias. Free Radic Biol Med. 2006;41(10):1557-1565.

27. Herold S, Exner M, Nauser T. Kinetic and mechanistic studies of the NO-mediated oxidation of oxymyoglobin and oxyhemoglobin. Biochemistry. 2001;40(11):3385-3395.

28. Beckman JS, Koppenol WH. Nitric oxide, superoxide, and peroxynitrite: the good, the bad, and ugly. Am J Physiol. 1996;271(5 Pt 1):C1424-C1437.

29. Atochin DN, Huang PL. Role of endothelial nitric oxide in cerebrovascular regulation. Curr Pharm Biotechnol. 2011;12(9):1334-1342.

30. Sehba FA, Schwartz AY, Chereshnev I, Bederson JB. Acute decrease in cerebral nitric oxide levels after subarachnoid hemorrhage. JCereb Blood Flow Metab. 2000;20(3):604-611.

31. Kanamaru K, Waga S, Kojima T, Fujimoto K, Niwa S. Endothelium-dependent relaxation of canine basilar arteries. Part 2: inhibition by hemoglobin and cerebrospinal fluid from patients with aneurysmal subarachnoid hemorrhage. Stroke. 1987;18(5):938-943.

32. Byrne JV, Griffith TM, Edwards DH, Harrison TJ, Johnston KR. Investigation of the vasoconstrictor action of subarachnoid haemoglobin in the pig cerebral circulation in vivo. Br J Pharmacol. 1989;97(3):669-674.

33. Pluta RM, Dejam A, Grimes G, Gladwin MT, Oldfield EH. Nitrite infusions to prevent delayed cerebral vasospasm in a primate model of subarachnoid hemorrhage. JAMA. 2005;293(12):1477-1484.

34. Sabri M et al. Mechanisms of microthrombi formation after experimental subarachnoid hemorrhage. Neuroscience. 2012;224:26-37.

35. Moncada S, Radomski MW, Palmer RM. Endothelium-derived relaxing factor. Identification as nitric oxide and role in the control of vascular tone and platelet function. Biochem Pharmacol. 1988;37(13):2495-2501.

36. Radziwon-Balicka A, et al. Differential eNOSsignalling by platelet subpopulations regulates adhesion and aggregation. Cardiovasc Res. 2017;113(14):1719-1731.

37. Petzold GC, et al. Nitric oxide modulates spreading depolarization threshold in the human and rodent cortex. Stroke. 2008;39(4):1292-1299.

38. Dreier JP et al. Nitric oxide scavenging by hemoglobin or nitric oxide synthase inhibition by $\mathrm{N}$-nitro-L-arginine induces cortical spreading ischemia when $\mathrm{K}+$ is increased in the subarachnoid space. JCereb Blood Flow Metab. 1998;18(9):978-990.

39. Willenborg DO, Staykova M, Fordham S, O'Brien $\mathrm{N}$, Linares $\mathrm{D}$. The contribution of nitric oxide and interferon gamma to the regulation of the neuro-inflammation in experimental autoimmune encephalomyelitis. J Neuroimmunol. 2007;191(1-2):16-25.
40. Guzik TJ, Korbut R, Adamek-Guzik T. Nitric oxide and superoxide in inflammation and immune regulation. J Physiol Pharmacol. 2003;54(4):469-487.

41. Pluta RM, et al. Cerebral vasospasm following subarachnoid hemorrhage: time for a new world of thought. Neurol Res. 2009;31(2):151-158.

42. Andersen $\mathrm{CBF}$ et al. Structure of the haptoglobin-haemoglobin complex. Nature. 2012;489(7416):456.

43. Boretti FS, et al. Sequestration of extracellular hemoglobin within a haptoglobin complex decreases its hypertensive and oxidative effects in dogs and guinea pigs. JClin Invest. 2009;119(8):2271-2280.

44. Schaer CA et al. Mechanisms of haptoglobin protection against hemoglobin peroxidation triggered endothelial damage. Cell Death Differ. 2013;20(11):1569-1579.

45. Graw JA et al. Haptoglobin or hemopexin therapy prevents acute adverse effects of resuscitation after prolonged storage of red cells. Circulation. 2016;134(13):945-960.

46. Belcher JD, et al. Haptoglobin and hemopexin inhibit vaso-occlusion and inflammation in murine sickle cell disease: Role of heme oxygenase-1 induction. PLOS ONE. 2018;13(4):e0196455.

47. Galea J, et al. The intrathecal CD163haptoglobin-hemoglobin scavenging system in subarachnoid hemorrhage. J Neurochem. 2012;121(5):785-792.

48. Baek JH, Zhang X, Williams MC, Schaer DJ, Buehler PW, D’Agnillo F. Extracellular Hb enhances cardiac toxicity in endotoxemic guinea pigs: protective role of haptoglobin. Toxins (Basel). 2014;6(4):1244-1259.

49. Whish S et al. The inner CSF-brain barrier: developmentally controlled access to the brain via intercellular junctions. Front Neurosci. 2015;9:16.

50. Brøchner CB, Holst CB, Møllgård K. Outer brain barriers in rat and human development. Front Neurosci. 2015;9:75.

51. Wang X, Mori T, Sumii T, Lo EH. Hemoglobininduced cytotoxicity in rat cerebral cortical neurons: caspase activation and oxidative stress. Stroke. 2002;33(7):1882-1888.

52. Pluta RM. Delayed cerebral vasospasm and nitric oxide: review, new hypothesis, and proposed treatment. Pharmacol Ther. 2005;105(1):23-56.

53. Hamel E. Perivascular nerves and the regulation of cerebrovascular tone. J Appl Physiol. 2006;100(3):1059-1064.

54. Iadecola C. The neurovascular unit coming of age: a journey through neurovascular coupling in health and disease. Neuron. 2017;96(1):17-42.

55. Pluta RM, Thompson BG, Dawson TM, Snyder SH, Boock RJ, Oldfield EH. Loss of nitric oxide synthase immunoreactivity in cerebral vasospasm. J Neurosurg. 1996;84(4):648-654.

56. Regan RF, Panter SS. Neurotoxicity of hemoglobin in cortical cell culture. Neurosci Lett. 1993;153(2):219-222.

57. Jung CS, Iuliano BA, Harvey-White J, Others. CSF Levels of ADMA, an endogenous inhibitor of endothelial nitric oxide synthase, are associated with cerebral vasospasm in a primate model of subarachnoid hemorrhage. J Neurosurg. 
2004;101(5):836-842.

58. Pyne-Geithman GJ, et al. Bilirubin production and oxidation in CSF of patients with cerebral vasospasm after subarachnoid hemorrhage. J Cereb Blood Flow Metab. 2005;25(8):1070-1077.

59. Clark JF, Reilly M, Sharp FR. Oxidation of bilirubin produces compounds that cause prolonged vasospasm of rat cerebral vessels: a contributor to subarachnoid hemorrhage-induced vasospasm. JCereb Blood Flow Metab. 2002;22(4):472-478.

60 . Joerk A, et al. Propentdyopents as heme degradation intermediates constrict mouse cerebral arterioles and are present in the cerebrospinal fluid of patients with subarachnoid hemorrhage. Circ Res. 2019;124(12):e101-e114.

61. Clark JF, Sharp FR. Bilirubin oxidation products (BOXes) and their role in cerebral vasospasm after subarachnoid hemorrhage. JCereb Blood Flow Metab. 2006;26(10):1223-1233.

62. Tang XD, Xu R, Reynolds MF, Garcia ML, Heinemann SH, Hoshi T. Haem can bind to and inhibit mammalian calcium-dependent Slo1 BK channels. Nature. 2003;425(6957):531-535.

63. Lin G, Macdonald RL, Marton LS, Kowalczuk A, Solenski NJ, Weir BK. Hemoglobin increases endothelin-1 in endothelial cells by decreasing nitric oxide. Biochem Biophys Res Commun. 2001;280(3):824-830.

64. Machi T, Stewart D, Kassell NF, Comair Y, Nihei $\mathrm{H}$, Tanaka Y. Hemoglobin enhances release of endothelin from cultured bovine endothelial cells. In: Sano K, Takakura K, Kassell NF, Sasaki T, eds. Cerbral Vasospasm. Tokyo, Japan: University of Tokyo Press; 1990:262-265.

65. Jahromi BS et al. Voltage-gated $\mathrm{K}+$ channel dysfunction in myocytes from a dog model of subarachnoid hemorrhage. JCereb Blood Flow Metab. 2008;28(4):797-811.

66. Ishiguro M, Wellman TL, Honda A, Russell SR, Tranmer BI, Wellman GC. Emergence of a R-type Ca2+ channel (CaV 2.3) contributes to cerebral artery constriction after subarachnoid hemorrhage. Circ Res. 2005;96(4):419-426.

67. Link TE, Murakami K, Beem-Miller M, Tranmer BI, Wellman GC. Oxyhemoglobin-induced expression of R-type $\mathrm{Ca} 2+$ channels in cerebral arteries. Stroke. 2008;39(7):2122-2128.

68. de Oliveira Manoel AL, Macdonald RL. Neuroinflammation as a target for intervention in subarachnoid hemorrhage. Front Neurol. 2018;9:292.

69. Cooper CE et al. Haptoglobin binding stabilizes hemoglobin ferryl iron and the globin radical on tyrosine $\beta 145$. Antioxid Redox Signal. 2013;18(17):2264-2273.

70. Vallelian F, et al. Spin trapping combined with quantitative mass spectrometry defines free radical redistribution within the oxidized hemoglobin:haptoglobin complex. Free Radic Biol Med. 2015;85:259-268.

71. Buehler PW, et al. Haptoglobin preserves the CD163 hemoglobin scavenger pathway by shielding hemoglobin from peroxidative modification. Blood. 2009;113(11):2578-2586.

72. Pimenova T, Pereira CP, Gehrig P, Buehler PW, Schaer DJ, Zenobi R. Quantitative mass spectrometry defines an oxidative hotspot in hemoglobin that is specifically protected by haptoglobin.
J Proteome Res. 2010;9(8):4061-4070.

73. Deuel JW, Vallelian F, Schaer CA, Puglia M, Buehler PW, Schaer DJ. Different target specificities of haptoglobin and hemopexin define a sequential protection system against vascular hemoglobin toxicity. Free Radic Biol Med. 2015;89:931-943.

74. Schaer DJ, Vinchi F, Ingoglia G, Tolosano E, Buehler PW. Haptoglobin, hemopexin, and related defense pathways-basic science, clinical perspectives, and drug development. Front Physiol. 2014;5:415.

75. Baek JH, et al. Iron accelerates hemoglobin oxidation increasing mortality in vascular diseased guinea pigs following transfusion of stored blood. JCI Insight. 2017;2(9):93577.

76. Sehba FA, Hou J, Pluta RM, Zhang JH. The importance of early brain injury after subarachnoid hemorrhage. Prog Neurobiol. 2012;97(1):14-37.

77. Suzuki H, Nakano F. To improve translational research in subarachnoid hemorrhage. Transl Stroke Res. 2018;9(1):1-3.

78. Gaastra B et al. Haptoglobin genotype and aneurysmal subarachnoid hemorrhage: Individual patient data analysis [published online ahead of print: April 5, 2019]. Neurology. https://doi. org/10.1212/WNL.0000000000007397.

79. Lipiski M, Deuel JW, Baek JH, Engelsberger WR, Buehler PW, Schaer DJ. Human Hp1-1 and Hp2-2 phenotype-specific haptoglobin therapeutics are both effective in vitro and in guinea pigs to attenuate hemoglobin toxicity. Antioxid Redox Signal. 2013;19(14):1619-1633.

80. Kristiansen $\mathrm{M}$ et al. Identification of the haemoglobin scavenger receptor. Nature. 2001;409(6817):198-201.

81. Schaer CA, Schoedon G, Imhof A, Kurrer MO, Schaer DJ. Constitutive endocytosis of CD163 mediates hemoglobin-heme uptake and determines the noninflammatory and protective transcriptional response of macrophages to hemoglobin. Circ Res. 2006;99(9):943-950.

82. Whitmore RG, Grant RA, LeRoux P, El-Falaki O, Stein SC. How large is the typical subarachnoid hemorrhage? A review of current neurosurgical knowledge. World Neurosurg. 2012;77(5-6):686-697.

83. Pluta RM, Butman JA, Schatlo B, Johnson DL, Oldfield EH. Subarachnoid hemorrhage and the distribution of drugs delivered into the cerebrospinal fluid. Laboratory investigation. J Neurosurg. 2009;111(5):1001-1004.

84. Connolly ES Jr et al. Guidelines for the management of aneurysmal subarachnoid hemorrhage: a guideline for healthcare professionals from the American Heart Association/american Stroke Association. Stroke. 2012;43(6):1711-1737.

85. Steiner T et al. European Stroke Organization guidelines for the management of intracranial aneurysms and subarachnoid haemorrhage. Cerebrovasc Dis. 2013;35(2):93-112.

86. Shen J, Pan JW, Fan ZX, Xiong XX, Zhan RY. Dissociation of vasospasm-related morbidity and outcomes in patients with aneurysmal subarachnoid hemorrhage treated with clazosentan: a meta-analysis of randomized controlled trials. J Neurosurg. 2013;119(1):180-189.
87. Macdonald RL, et al. Clazosentan, an endothelin receptor antagonist, in patients with aneurysmal subarachnoid haemorrhage undergoing surgical clipping: a randomised, double-blind, placebocontrolled phase 3 trial (CONSCIOUS-2). Lancet Neurol. 2011;10(7):618-625.

88. Hansen-Schwartz J, Vajkoczy P, Macdonald RL, Pluta RM, Zhang JH. Cerebral vasospasm: looking beyond vasoconstriction. Trends Pharmacol Sci. 2007;28(6):252-256.

89. Fathi AR, Bakhtian KD, Pluta RM. The role of nitric oxide donors in treating cerebral vasospasm after subarachnoid hemorrhage. Acta Neurochir Suppl. 2011;110(Pt 1):93-97.

90. Ehlert A, Manthei G, Hesselmann V, Mathias K, Bein B, Pluta R. A case of hyperacute onset of vasospasm after aneurysmal subarachnoid hemorrhage and refractory vasospasm treated with intravenous and intraventricular nitric oxide: a mini review. World Neurosurg. 2016;91:673.e11-673.e18.

91. Ehlert A, et al. Molsidomine for the prevention of vasospasm-related delayed ischemic neurological deficits and delayed brain infarction and the improvement of clinical outcome after subarachnoid hemorrhage: a single-center clinical observational study. J Neurosurg. 2016;124(1):51-58.

92. Terpolilli NA, et al. Nitric oxide inhalation reduces brain damage, prevents mortality, and improves neurological outcome after subarachnoid hemorrhage by resolving early pial microvasospasms. J Cereb Blood Flow Metab. 2016;36(12):2096-2107.

93. Gladwin MT et al. The emerging biology of the nitrite anion. Nat Chem Biol. 2005;1(6):308-314.

94. Oldfield EH, et al. Safety and pharmacokinetics of sodium nitrite in patients with subarachnoid hemorrhage: a phase IIa study. J Neurosurg. 2013;119(3):634-641.

95. Fathi AR, Pluta RM, Bakhtian KD, Qi M, Lonser $R R$. Reversal of cerebral vasospasm via intravenous sodium nitrite after subarachnoid hemorrhage in primates. JNeurosurg. 2011;115(6):1213-1220.

96. Buehler PW, Butt OI, D’Agnillo F. Sodium nitrite induces acute central nervous system toxicity in guinea pigs exposed to systemic cell-free hemoglobin. Biochem Biophys Res Commun. 2011;409(3):412-417.

97. Elmer J, Harris DR, Sun G, Palmer AF. Purification of hemoglobin by tangential flow filtration with diafiltration. Biotechnol Prog. 2009;25(5):1402-1410.

98. Pimenova T, Pereira CP, Schaer DJ, Zenobi R. Characterization of high molecular weight multimeric states of human haptoglobin and hemoglobin-based oxygen carriers by high-mass MALDI MS. JSep Sci. 2009;32(8):1224-1230.

99. Zhou Y, Jia Y, Buehler PW, Chen G, Cabrales P, Palmer AF. Synthesis, biophysical properties, and oxygenation potential of variable molecular weight glutaraldehyde-polymerized bovine hemoglobins with low and high oxygen affinity. Biotechnol Prog. 2011;27(4):1172-1184.

100.Herold S. Kinetic and spectroscopic characterization of an intermediate peroxynitrite complex in the nitrogen monoxide induced oxidation of oxyhemoglobin. FEBS Lett. 1999;443(1):81-84. 